\title{
MODOS DE VIDA DE LOS CAZADORES-RECOLECTORES DE LA COSTA ARREICA DEL NORTE GRANDE DE CHILE: UNA APROXIMACIÓN BIOARQUEOLÓGICA A LAS POBLACIONES PREHISTÓRICAS DE TALTAL ${ }^{1}$
}

\section{LIFESTYLES OF HUNTERS-GATHERERS FROM THE INTERFLUVIC COAST OF NORTHERN CHILE: A BIOARCHAEOLOGICAL APPROACH TO THE PREHISTORIC POPULATIONS OF TALTAL}

\author{
Pedro Andrade ${ }^{1}$, Diego Salazar ${ }^{2}$, Josefina Urrea ${ }^{1}$ y Victoria Castro ${ }^{3}$
}

\begin{abstract}
Se presentan los resultados obtenidos de análisis bioarqueológicos realizados en 36 individuos provenientes de 25 sitios prehispánicos de la costa de Taltal, los cuales muestran la continuidad en la zona del modo de vida cazador-recolector-pescador, asociado a la explotación y consumo de recursos marinos. Se especifican una serie de actividades que estos individuos habrían llevado a cabo, a partir de la presencia de enfermedades articulares degenerativas y algunos marcadores de estrés ocupacional, además de presentarse evidencia de condiciones paleopatológicas metabólicas e infecciosas. Al mismo tiempo, se establecen comparaciones con poblaciones prehistóricas con modos de vidas similares que habitaron ambientes costeros del extremo norte y norte semiárido de Chile, para finalmente reflexionar en torno a las particularidades del área de estudio.
\end{abstract}

Palabras claves: bioarqueología, costa de Taltal, cazadores-recolectores-pescadores, modos de vida.

This study presents the results of the bioarchaeological analyses of 36 individuals from the coastal area of Taltal. These results indicate that these individuals had a hunter-gatherer-fisher lifestyle, related to the exploitation and consumption of marine resources. A series of activities performed by these individuals is proposed, based on the presence of articular joint diseases and some markers of occupational stress. Also, evidence of metabolic and infectious diseases is presented. At the same time, comparisons are made with other prehistoric populations with similar lifestyles that inhabited coastal environments in the extreme North and semiarid North of Chile. Finally, some reflections about the study area are presented.

Key words: Bioarchaeology, coastal area of Taltal, hunter-gatherer-fisher, lifestyles.

El cuerpo humano se presenta como un interesante rasgo, dada su particular condición de interfase entre los dominios biológicos y sociales (Meskell 1998:158; Sofaer 2006:55-61). El análisis de los modos de vida es una gran oportunidad para unir ambas dimensiones, dado que no solamente dan cuenta de aspectos biológicos, sino que también de actividades sociales características de una población. Esta aproximación permite comprender el significado implícito de las actividades pasadas desde un punto de vista holístico, dado que el cuerpo es una creación física y fenomenológica, pero también un producto cultural (Synnott 1993:4), donde convergen los aspectos privados y públicos de una sociedad (Joyce 2005:142).
En este contexto, el propósito del presente trabajo es presentar una visión bioarqueológica exploratoria de los restos óseos humanos provenientes de distintos sitios prehispánicos de la costa de Taltal ${ }^{2}$. Los análisis presentados se orientan a la reconstrucción de los modos de vida de las antiguas poblaciones, con el objeto de complementar la escasa información arqueológica disponible para una zona que se presenta como un vacío investigativo entre las costas de Valles Occidentales y el Norte Semiárido de Chile (Figura 1).

La importancia de este estudio exploratorio radica en que los antecedentes que se conocen para la prehistoria de Taltal (Bird 1943; Capdeville 1921, 1922, 1923; Castelleti 2007; Castelleti et al.

\footnotetext{
1 Departamento de Sociología y Antropología, Universidad de Concepción, Concepción, Chile. pandradem@udec.cl; jourrea@udec.cl

2 Departamento de Antropología, Universidad de Chile, Santiago, Chile. dsalazar@uchile.cl

3 Departamento de Antropología, Universidad Alberto Hurtado, Santiago, Chile. vcastror53@ gmail.com
} 


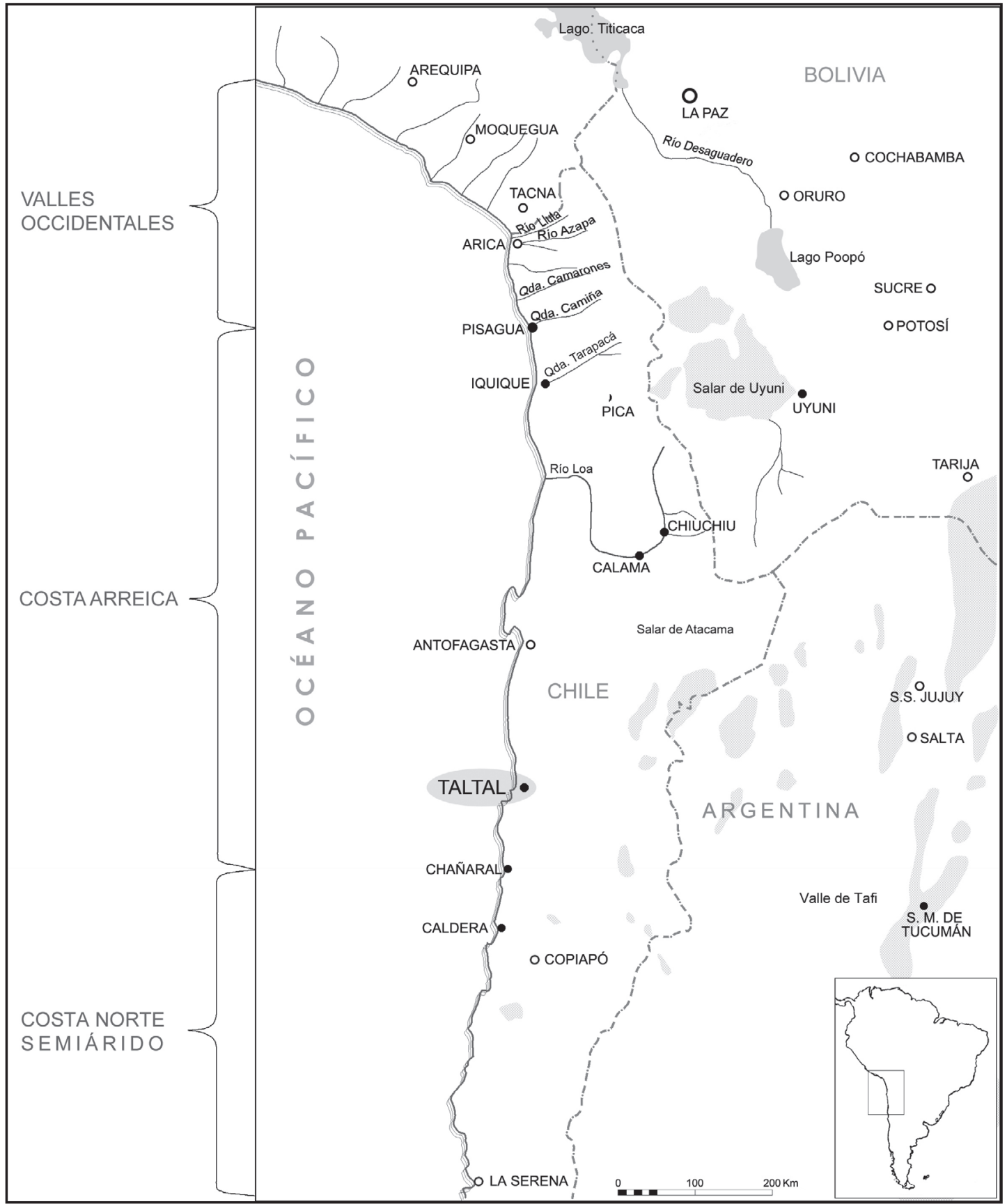

Figura 1. Ubicación de la zona de estudio.

Location of the research area.

2010; Castelleti y Maltrain 2010; Contreras et al. 2008; Durán 1985; Llagostera 1989; Núñez 1984; Salazar et al. 2009; Silva y Bahamondes 1969) señalan una continuidad histórica del modo de vida cazador-recolector de adaptación marina en el área, en contraste con lo que sucede en regiones más septentrionales y meridionales donde los procesos formativos absorbieron y transformaron las formas de vida arcaicas (Castillo 1986; Kuzmanic y Castillo 1986; Muñoz y Chacama 2012; Núñez y Santoro 2011). No obstante, hasta la fecha la hipótesis de la continuidad del modo de vida arcaico no ha 
sido calibrada sistemáticamente desde la evidencia bioantropológica. Es así como en el presente trabajo nos proponemos evaluar esta hipótesis así como explorar las características particulares del modo de vida local, por medio del análisis bioarqueológico de 36 individuos excavados en distintos sitios de la localidad y correspondientes a diferentes momentos cronológicos que van desde el Arcaico Tardío (ca. 4.500 cal a.p.) hasta el período Alfarero Tardío (ca. 700 cal a.p.). El rango cronológico que abarca la muestra corresponde justamente al momento en que las poblaciones de Valles Occidentales y del Norte Semiárido transforman el modo de vida arcaico al integrarse a procesos formativos que introdujeron importantes transformaciones tecnológicas, económicas y sociales (Castillo 1986; Muñoz 1989; Muñoz y Chacama 2012; Niemeyer 1997; Núñez y Santoro 2011; Troncoso y Pavlovic 2013).

La muestra analizada comprende tanto a individuos que fueron recuperados de excavaciones controladas y sistemáticas, como restos de individuos saqueados y disturbados que fueron recuperados por arqueólogos profesionales desde la superficie. Pese a esta diferencia, la totalidad de los individuos provienen de entierros aislados, generalmente dentro de aleros o junto a bloques rocosos, patrón funerario dominante en el área luego del abandono de las estructuras del patrón Caleta Huelén 42 -hecho ocurrido aproximadamente hacia el 4.500 cal a.p.- y hasta el Período Intermedio Tardío (Salazar 2013). Los sitios estudiados se ubican en un segmento de cerca de 100 kilómetros de costa, entre Punta Loreto y la desembocadura de la Quebrada El Bronce (Figura 2).

Cabe señalar que solo el 52,78\% de la muestra se encuentra asignada a un momento cronológico específico (Tabla 1), ya sea por fechación directa del esqueleto $(36,11 \%)$ o bien por las evidencias contextuales que lo acompañaban como ofrenda o ajuar (16,67\%), mientras que el 47,22\% de la muestra no ha podido ser asignado a un momento cronológico preciso. No obstante, y si bien es evidente que los restos humanos sin contextos arqueológicos claros presentan limitaciones en términos interpretativos, también es cierto que han probado ser de enorme utilidad para enfrentar problemas bioarqueológicos y biomédicos de las poblaciones pasadas (Arriaza et al. 2008), por lo que han sido incorporados en este trabajo como una muestra de control que permite explorar si los patrones identificados en los restos óseos excavados en forma sistemática y/o adecuadamente cronologizados, se reconocen también en una muestra mayor proveniente del área de estudio y situada posiblemente dentro del mismo rango cronológico antes mencionado (ca. 4.500-700 cal a.p.) a juzgar por el tipo de sitio donde se recuperaron los restos esqueletales.

\section{Arqueología de la Costa de Taltal}

Las primeras secuencias cronológicas para la costa de Taltal fueron propuestas por destacados investigadores de la primera mitad del siglo XX como el propio Max Uhle, Augusto Capdeville y Junius Bird (Bird 1943, 1946; Mostny 1964), pero serían trabajos más recientes, realizados a partir del análisis de colecciones y de estudios de impacto ambiental, los que permitirían darle una mayor precisión cronológica a la secuencia cultural de Taltal, a partir de una correlación con contextos bien datados en otras zonas (Berdichewski 1962; Durán 1985, Núñez 1984), así como de una batería de fechados para diversos sitios ubicados entre quebrada El Bronce y Zapatero (Andrade y Salazar 2011; Castelleti 2007; Contreras et al. 2008 y 2011; Salazar 2013).

A partir de estos antecedentes, hoy sabemos que las primeras ocupaciones de la costa de Taltal corresponden al Complejo Huentelauquén y han sido identificadas en torno a los 11.000 cal a.p. en el Alero Cascabeles (Castelleti 2007; Castelleti et al. 2010) y, más recientemente, en San Ramón 15 (Salazar et al. 2011) y en los Aleros 224A y 225 (Salazar et al. 2013). Se trataría de las primeras adaptaciones marítimas de la costa norte (Llagostera 2005; Sandweiss 2008) correspondientes a grupos cazadores-recolectores costeros de amplia movilidad en sentido norte-sur, que en la zona de Taltal se complementó con sistemáticos accesos hacia la pampa intermedia con el objeto de obtener materias primas líticas y minerales (Castelleti 2007; Salazar et al. 2013). Con posterioridad a estas ocupaciones, se produce un "silencio arqueológico" de cerca de 2.000 años en la zona, hecho que también se ha reportado a nivel de toda la costa arreica (Llagostera 2005). Para el Arcaico Medio de Taltal (ca. 8.0005.500 cal a.p.) se conoce una mayor cantidad de sitios, incluyendo los conchales "clásicos" de la zona. Estos sitios han sido considerados como campamentos residenciales con cierta estabilidad de ocupación (Andrade y Salazar 2011; Castelleti 2007; Salazar 2013), siendo los más conocidos los 


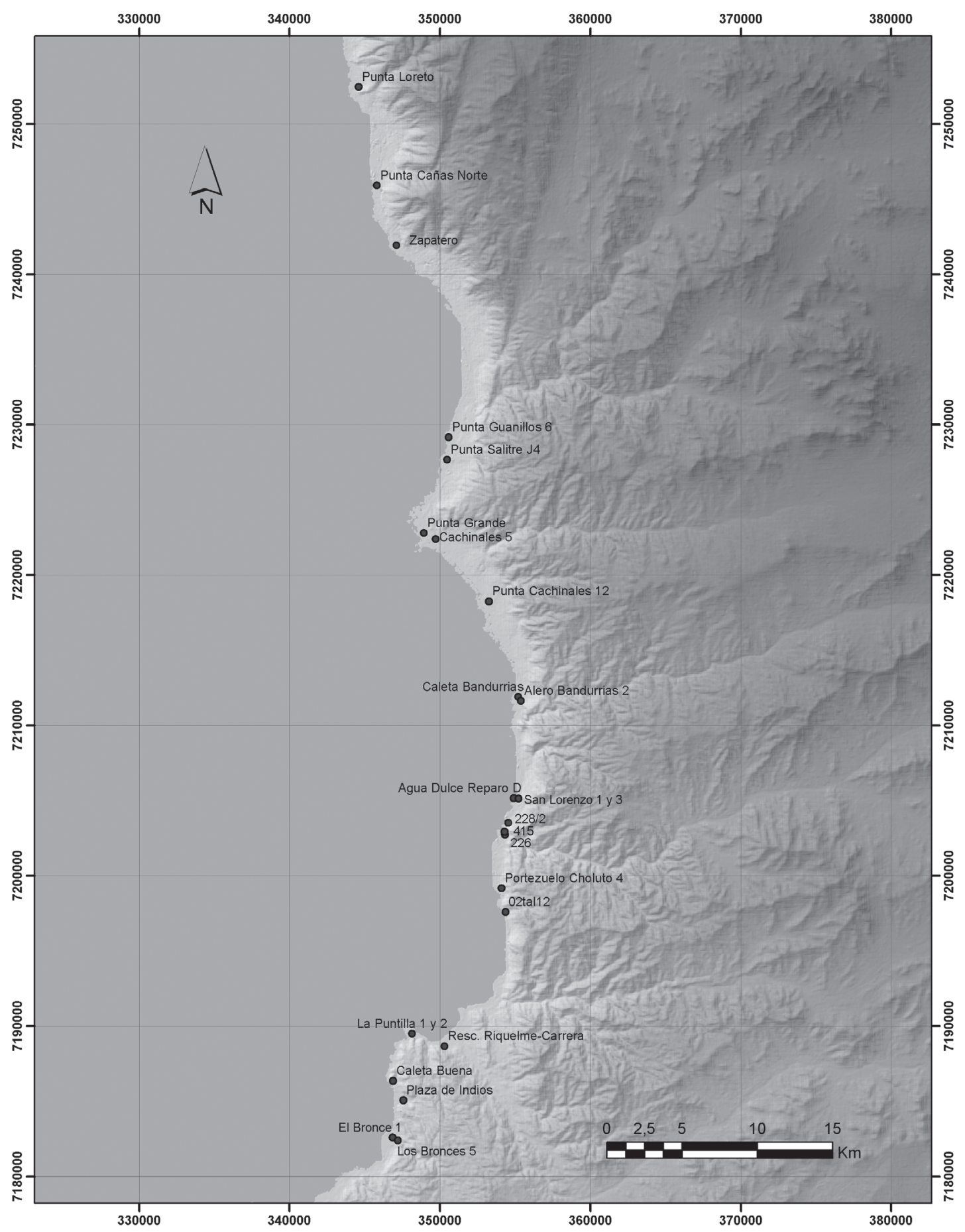

Figura 2. Ubicación de los sitios de donde fueron recuperados los individuos analizados. Location of archaeological sites from which the analyzed individuals were recovered.

de Morro Colorado (Bird 1943; Capdeville 1921), Punta Morada (Bird 1943; Capdeville 1921), Las Conchas (Silva y Bahamondes 1969), Punta Grande
(Durán 1985) y Zapatero (Salazar 2013). La cultura material y el tipo de sitio que caracterizan a la ocupación del Arcaico Medio indican un modo 
Tabla 1. Sitios y cantidad mínima de individuos analizados en nuestra muestra.

Sites and Minimum Number of Individuals (MNI) of the analyzed sample.

\begin{tabular}{|c|c|c|c|c|}
\hline $\begin{array}{l}\text { Número } \\
\text { correlativo }\end{array}$ & Sitio & $\begin{array}{l}\text { Número } \\
\text { mínimo de } \\
\text { individuos }\end{array}$ & Cronología & Referencia \\
\hline 2 & San Lorenzo 1 & 1 & Arcaico Tardío (ca. 4.000 a.p.) & FONDECYT 1080666 \\
\hline 3 & Punta Loreto & 1 & Alfarero Tardío (ca. 900 a.p.) & FONDECYT 1080666 \\
\hline 4 & Caleta Buena & 1 & Alfarero Temprano (ca. 1.500 a.p.) & FONDECYT 1080666 \\
\hline 7 & La Puntilla 1 & 1 & No Determinado & Museo A. Capdeville Taltal \\
\hline 8 & La Puntilla 2 & 1 & No Determinado & Museo A. Capdeville Taltal \\
\hline 9 & Los Bronces 5 & 1 & Alfarero Temprano & Museo A. Capdeville Taltal \\
\hline 10 & Plaza de Indios & 1 & Alfarero Temprano & Museo A. Capdeville Taltal \\
\hline 11 & Rescate Riquelme con Carrera & 1 & No Determinado & Museo A. Capdeville Taltal \\
\hline 12 & Cachinales 5 & 3 & No Determinado & FONDECYT 1080666 \\
\hline 17 & Caleta Bandurrias & 1 & No Determinado & FONDECYT 1080666 \\
\hline 18 & Agua Dulce Reparo D & 6 & No determinado & FONDECYT 1080666 \\
\hline 19 & Portezuelo Choluto 4 & 1 & Alfarero Temprano (ca. 2.500 a.p.) & Castelleti 2007 \\
\hline 20 & $226-5$ & 1 & Alfarero Tardío & Castelleti 2007 \\
\hline 21 & $228-230$ & 1 & Alfarero Tardío & Castelleti 2007 \\
\hline 22 & 415 Alero 1 & 2 & Alfarero Tardío & Castelleti 2007 \\
\hline 23 & $02 \mathrm{Ta} 012$ & 1 & No Determinado & Gaete 2001 \\
\hline 24 & Zapatero & 1 & Alfarero Tardío (ca. 1.300 a.p.) & FONDECYT 1110196 \\
\hline 25 & El Bronce 1 & 1 & No Determinado & Museo de Antofagasta \\
\hline
\end{tabular}

de vida cazador-recolector de adaptación marítima especializada, con circuitos de movilidad residencial posiblemente más reducidos que durante el Arcaico Temprano, pero que no obstante incluyó desplazamientos logísticos longitudinales hacia el interior de la Cordillera de la Costa (Andrade y Salazar 2011; Castelleti 2007; Salazar 2013).

No se han reportado enterratorios para el Arcaico Temprano y Medio de Taltal, lo que plantea dudas respecto de la estabilidad de estas ocupaciones en el área y hace por el momento imposible explorar el tipo de adaptación de estas poblaciones desde el registro bioantropológico.

Para la primera mitad del Arcaico Tardío (ca. 5.500-4.500 cal a.p.), la cantidad de sitios en la costa de Taltal aumenta considerablemente, lo que lleva a Castelleti (2007) a postular un clímax de la ocupación arcaica de la zona. Corresponde a lo que Capdeville (1921) denominó "Civilización Dolménica" de la "Gente de los Círculos de Piedra". Si bien semejanzas ergológicas y, en algunos casos, en los patrones mortuorios, ha llevado a algunos investigadores a incluir estos contextos dentro de la Tradición Chinchorro (Contreras y Núñez 2009; Núñez 2003; Rivera 2002), o bien dentro del “Complejo Quiani”" (Llagostera 1989), la presencia durante este período de arquitectura doméstica y numerosos entierros asociados a las viviendas permite pensar que estamos ante poblaciones locales de movilidad más restringida, características de la costa arreica entre Caleta Huelén y Taltal o quizás aún más al sur (Ballester y Gallardo 2011). Los contextos funerarios correspondientes a este Período son frecuentes en el área, a lo menos a partir de los registros de Capdeville y hallazgos más recientes (p.ej., Contreras et al. 2008; Llagostera y Llagostera 2010). Con posterioridad al 4.500 cal a.p. se habría mantenido el modo de vida cazador-recolector de adaptación marítima, no obstante se habrían abandonado los principales sitios con estructuras del tipo Caleta Huelén 42 que caracterizaron a la primera mitad del Arcaico Tardío en la zona (Castelleti 2007). Las ocupaciones humanas entre este momento y hasta la llegada de los incas a la zona parece haber estado caracterizada por una mayor movilidad residencial materializada en campamentos habitacionales más transitorios y con viviendas livianas (Salazar et al. 2009), en forma similar al modo de vida reportado por la información histórica (Ballester et al. 2010; 
Ballester y Gallardo 2011; Bittmann 1979). Si bien no existe consenso acerca de los momentos específicos de este cambio en el patrón de asentamiento (compárese por ejemplo Castelleti 2007 y Salazar et al. 2009), sí existe acuerdo en torno al hecho de que las comunidades locales experimentaron transformaciones radicales en sus sistemas de organización territorial, pero sin abandonar la economía marítima. El estudio que presentamos nos permite complementar investigaciones anteriores al evaluar desde el registro bioarqueológico las implicancias que tuvieron las transformaciones de los sistemas de asentamiento sobre un modo de vida que mantuvo una economía de subsistencia basada en la explotación y consumo casi exclusivamente de recursos litorales y marinos. En el marco de estudios de impacto ambiental, excavaciones de rescate y nuestros proyectos de investigación en el área, se han exhumado una serie de individuos correspondientes a este momento cronológico (ca. 4.500-700 cal a.p.), los que constituyen la base del presente trabajo.

\section{Estudios Bioarqueológicos y Modo de Vida en la Costa Arreica del Norte Grande de Chile}

Dentro de la costa desértica del Norte Grande de Chile existen abundantes estudios sobre restos bioarqueológicos, específicamente en la subárea Valles Occidentales entre Arica y Pisagua (cf. Alfonso et al. 2007; Arriaza et al. 1995; Arriaza 1993; Aufderheide et al. 2004; Boston y Arriaza 2009; Costa-Junqueira, Varela et al. 2000; CostaJunqueira, Cocilovo et al. 2000; Cocilovo et al. 1999; Henríquez y Arriaza 2013; Quevedo y Cocilovo 1995; Standen y Arriaza 2000, entre otros). Lamentablemente esta situación no se ve reflejada en la zona de la costa arreica, entendiéndose esta según la definición de Llagostera (1989) entre las localidades de Pisagua y Chañaral. Destacan en esta zona los estudios de Cocilovo y colaboradores (2005), Llagostera y Llagostera (2010), Ardiles y colaboradores (2011) y Arias y Herrera (2012), quienes estudiaron muestras esqueletales de los sitios Caleta Huelén 42, Los Bronces 1, Punta Blanca/Chacaya y Cáñamo 3, respectivamente. Los Bronces 1 sería el único sitio correspondiente a la zona de Taltal, pero el estudio de Llagostera y Llagostera se basó en un solo individuo subadulto, por lo que presenta escasos antecedentes en términos de indicadores de modos de vida.
Dada la escasez de estudios bioarqueológicos publicados para la costa arreica, el presente trabajo constituye una primera aproximación que permite comenzar a llenar este importante vacío investigativo para la zona de Taltal y Paposo. Continuar con este tipo de estudios resulta fundamental, puesto que las diversas investigaciones realizadas en la zona de Valles Occidentales han mostrado importantes cambios a nivel bioarqueológico entre el Arcaico y los períodos agroalfareros, como un aumento durante el Formativo de caries, pérdida de piezas dentales antemortem, hipoplasia del esmalte dental (Alfonso et al. 2007), así como lesiones degenerativas articulares en la columna vertebral como DISH y espondiloartropatías seronegativas (Arriaza 1993), un leve aumento de evidencias de tuberculosis (Arriaza et al. 1995), así como la aparición de dietas mixtas agromarítimas (Aufderheide et al. 2002; véase Petrucelli et al. 2012 y Roberts et al. 2013 para resultados distintos). Asimismo, en comparación con el período Arcaico, a contar del Formativo en los Valles Occidentales se advertiría una disminución de las expresiones de treponematosis (Standen y Arriaza 2000), de nódulos de Schmorl (Henríquez y Arriaza 2013) y de la intensidad del desgaste oclusal (Alfonso et al. 2007), presentándose también variaciones entre sitios del interior y de la costa justamente debido al impacto diferencial de la economía agrícola y los nuevos patrones de asentamiento sobre la población costera de raigambre arcaica (Alfonso et al. 2007; Watson et al. 2013). En consecuencia, resulta relevante explorar el contexto bioarqueológico de aquellas poblaciones de cazadores recolectores costeros que no se incorporaron al proceso formativo del norte grande de Chile, tal como sucedió en la costa arreica de la actual II Región de Antofagasta. En este contexto, cabría esperar una mayor continuidad entre los modos de vida reflejados en la evidencia bioarqueológica para el Arcaico y los períodos Agroalfareros posteriores. No obstante, también es necesario considerar que la arqueología de la zona ha propuesto importantes cambios en el patrón de asentamiento a contar del $4.500 \mathrm{cal}$ a.p. en el área de estudio (Castelleti 2007; Salazar et al. 2009), por lo que resulta de importancia determinar si dichas transformaciones tuvieron o no injerencia sobre los modos de vida que revela la bioarqueología. 


\section{Material y Método}

Se trabajó con una muestra de 36 individuos provenientes de 25 sitios ubicados en la costa arreica de Taltal y abarcando un lapso cronológico de aproximadamente 4.000 años, entre fines del Arcaico Tardío y el Período Alfarero Tardío (Figura 2, Tablas 1 y 2). Solo se han incorporado a este estudio individuos adultos, ya que ellos representan de mejor manera el modo de vida de una población, tanto a nivel de patologías, como a nivel de marcadores de estrés ocupacional, debido a que las unidades óseas de los infantes se encuentran en formación y no se puede establecer de forma fehaciente cuándo comienzan a participar en las actividades productivas adultas (Lewis 2007).

Para la determinación de edad de la muestra analizada se consideraron las categorías generales de edad propuestas por Buikstra y Ubelaker (1994): adulto joven (21-35 años), adulto medio (36-50 años) y adulto mayor (51 y más). La determinación de edad fue estimada en la fusión de las epífisis de los huesos largos y de las vértebras sacras (Bass 1987, Buikstra y Ubelaker 1994, Campillo y Subirá 2004), el cierre de las suturas craneales (Hershkovitz et al. 1997), desarrollo osteológico de las unidades óseas
(Scheuer y Black 2000) y morfología del extremo esternal de la cuarta costilla (Iscan et al. 1984). La determinación de sexo fue realizada considerando los rasgos morfológicos observados en cráneo y coxales (Bass 1987; Buikstra y Ubelaker 1994; Campillo y Subirá 2004). La asignación de sexo se realizó en términos de cinco categorías: Femenino, probable Femenino, Sexo Indeterminado, probable Masculino y Masculino. El uso de estas cinco categorías se consideró oportuno dado que un $50 \%$ de los esqueletos están incompletos y/o fragmentados por lo que no presentan todos los rasgos necesarios para hacer una determinación de sexo precisa. La estimación de la estatura fue realizada a través de la medida de la longitud máxima de los huesos largos, usando la fórmula planteada por Genovés, modificada por Del Ángel y Cisneros (2002). Para poder determinar si las diferencias observadas en la estatura poseían un significado estadístico, se utilizó el test de $T$ de Student, con un intervalo de confianza del $95 \%$. En cuanto a la determinación del número mínimo de individuos (MNI), se utilizaron las recomendaciones planteadas por Adams y Koningsberg $(2004,2008)$. Para el reconocimiento de patologías, se consideraron todas aquellas

Tabla 2. Distribución de MNI, sexo y edad por sitio (MNI: Mínimo número de individuos, PF: Probable femenino, F: Femenino, PM: Probable masculino, M: Masculino, I: Indeterminado, AJ: Adulto Joven, AM: Adulto Medio, AEI: Adulto de Edad Indeterminada).

Distribution of MNI, sex and age by site (MNI: Minimum number of individuals, PF: Probable female, F: Female, PM: Probable Male, M: Male, I: Indeterminate; AJ: Young Adult; AM: Middle-Aged Adult; AEI: Adult of Unknown Age).

\begin{tabular}{|c|c|c|c|c|c|c|c|c|c|c|c|c|c|c|c|}
\hline Sitio & MNI & \multicolumn{2}{|r|}{ Sexo } & \multicolumn{2}{|r|}{ Edad } & Sitio & MNI & \multicolumn{5}{|c|}{ Sexo } & \multicolumn{3}{|c|}{ Edad } \\
\hline San Lorenzo 1 & 1 & $\mathrm{X}$ & & $X$ & & Punta Cañas Norte & & & $\mathrm{X}$ & & & & $\mathrm{X}$ & & \\
\hline Punta Loreto & 1 & & $\mathrm{X}$ & $\mathrm{X}$ & & & 3 & & & & $\mathrm{X}$ & & $\mathrm{X}$ & & \\
\hline Caleta Buena & 1 & $\mathrm{X}$ & & & $\mathrm{X}$ & Punta Guanillos 6 & 1 & & & & & $\mathrm{X}$ & $\mathrm{X}$ & & \\
\hline & 4 & $\mathrm{X}$ & & & $\mathrm{X}$ & Caleta Bandurrias & 1 & & & & & $\mathrm{X}$ & & & $\mathrm{X}$ \\
\hline & & & $\mathrm{X}$ & $\mathrm{X}$ & & & 4 & & & & & $\mathrm{X}$ & & & $\mathrm{X}$ \\
\hline Punta Grande & 1 & $\mathrm{X}$ & & & $\mathrm{X}$ & Agua Dulce Reparo D & & & & & & $\mathrm{X}$ & & & $\mathrm{X}$ \\
\hline La Puntilla 1 & 1 & & $\mathrm{X}$ & $\mathrm{X}$ & & & & & & & & $\mathrm{X}$ & & & $\mathrm{X}$ \\
\hline La Puntilla 2 & 1 & $\mathrm{X}$ & & $\mathrm{X}$ & & & & & & & & $\mathrm{X}$ & & & $\mathrm{X}$ \\
\hline & & & $\mathrm{X}$ & & $\mathrm{X}$ & $02 \mathrm{Ta} 012$ & 1 & & & & & $\mathrm{X}$ & $\mathrm{X}$ & & \\
\hline & 3 & & $\mathrm{X}$ & & $\mathrm{X}$ & El Bronce 1 & 1 & & & & & $\mathrm{X}$ & $\mathrm{X}$ & & \\
\hline \multirow[t]{2}{*}{ Punta Cachinales 12} & 1 & $\mathrm{X}$ & & $\mathrm{X}$ & & Zapatero & 1 & & $\mathrm{X}$ & & & & $\mathrm{X}$ & & \\
\hline & & & & & & Total & 36 & 1 & 12 & 0 & 5 & 18 & 17 & 8 & 11 \\
\hline
\end{tabular}


enfermedades degenerativas, infecciosas y metabólicas, siguiendo las indicaciones de Mann y Hunt (2005), Aufderheide y Rodríguez-Martín (1998) y Buikstra y Ubelaker (1994). En la determinación de retroversión del miembro superior se consideró su ausencia o presencia de acuerdo con los parámetros planteados por Rhodes y Churchill (2008).

Nuestros resultados fueron comparados y discutidos con estudios realizados previamente en poblaciones con un modo de vida similar, en regiones cercanas. Para esto, se privilegió la información bibliográfica disponible de los grupos humanos arcaicos que habitaron la zona de Arica, en los sitios de Acha 3, Morro 1, Morro 1-5, Morro 1-6, Camarones, Quiani y Playa Miller 8, trabajados por varios autores (Arriaza y Standen 2008; Costa-Junqueira, Cocilovo et al. 2000; Costa-Junqueira, Varela et al. 2000; Quevedo 1984; Standen y Arriaza 2000; Standen y Santoro 2004; entre otros) así como la evidencia disponible sobre el Arcaico Tardío del Norte Semiárido, específicamente proveniente de los sitios Punta Teatinos y El Cerrito (Quevedo 2000; Quevedo et al. 2003).

\section{Resultados}

\section{Determinación de MNI, sexo y edad}

Se trabajó con un MNI total de 36 individuos adultos. Estos se distribuyen en cinco individuos masculinos, 12 de sexo femenino, uno de sexo probablemente femenino, y 18 de sexo no determinado, estando la edad de los mismos concentrados principalmente en la categoría adulto joven y en menor medida en la categoría adulto medio, sin registrarse adultos mayores (Tabla 2).

\section{Estatura}

La muestra se ha visto limitada en cuanto a condiciones de asociaciones directas que nos permitan establecer determinaciones de sexo y edad. No obstante lo anterior, se pudo determinar, de manera preliminar, que esta tendría una estatura promedio que alcanzaría los 146,58 $\pm 4,07 \mathrm{~cm}$ en el caso de las mujeres, y una estatura promedio de $158,14 \pm 4,61 \mathrm{~cm}$ en el caso de los hombres (Figura 3).

\section{Enfermedades metabólicas y del crecimiento}

La presencia de enfermedades metabólicas, estuvo únicamente representada en la aparición de criba orbitaria e hiperostosis porótica. La muestra comprendió 11 cráneos susceptibles de ser analizados, tanto completos como fragmentados. De estos, se pudo observar tres con huellas de criba orbitaria ( $\mathrm{La}$ Puntilla Individuo 1, San Lorenzo 3 y Portezuelo Choluto 4), uno con huellas de hiperostosis porótica (Punta Loreto) y uno que poseía huellas de ambas patologías (La Puntilla Individuo 2), lo que

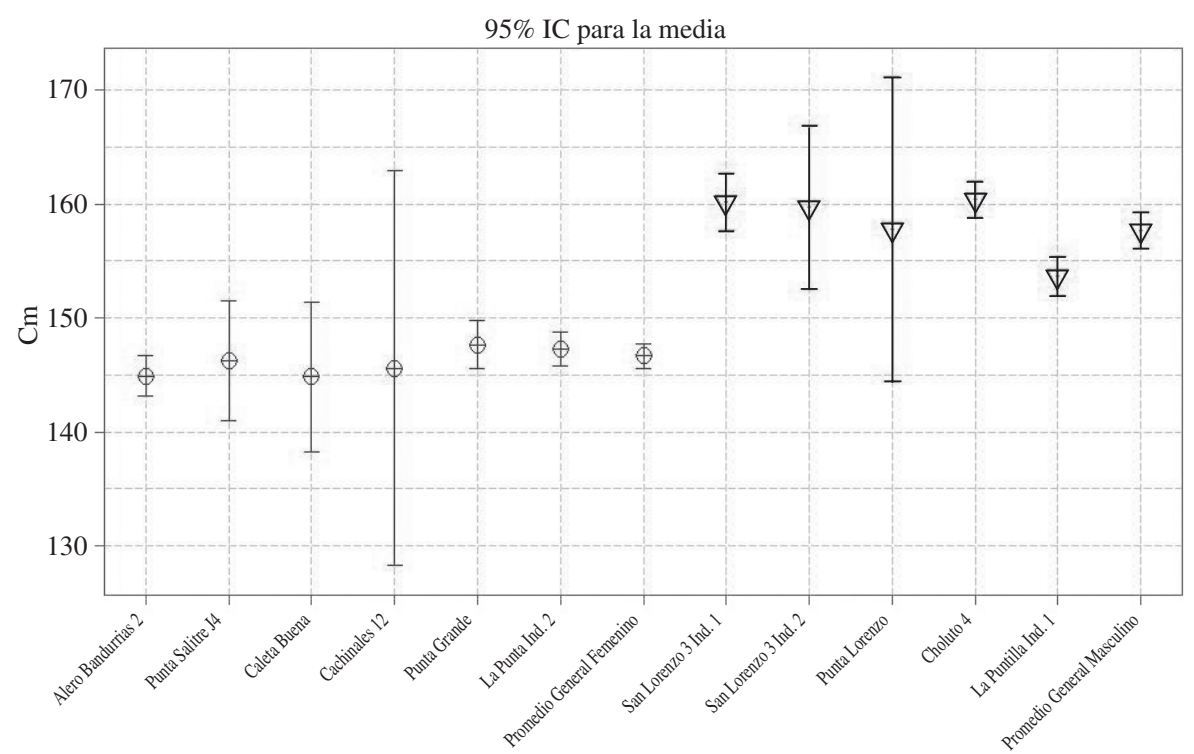

Figura 3. Distribución de estatura por individuo y sexo (gris: femenino; negro: masculino). Stature distribution by individual and sex (gray: feminine; black: masculine). 
Tabla 3. Presencia de criba orbitaria e hiperostosis porótica por individuo (D: Lado derecho, I: Lado derecho, NO: Número de órbitas, NPR: Número de parietales registrados; NP: No presente, NR: No posible de registrar).

Presence of cribra orbitalia and porotic hyperostosis by individual (D: Right side, I: Left side, NO: Number of orbits, NPR: Number of recorded parietals, NP: Not present, NR: Not recordable).

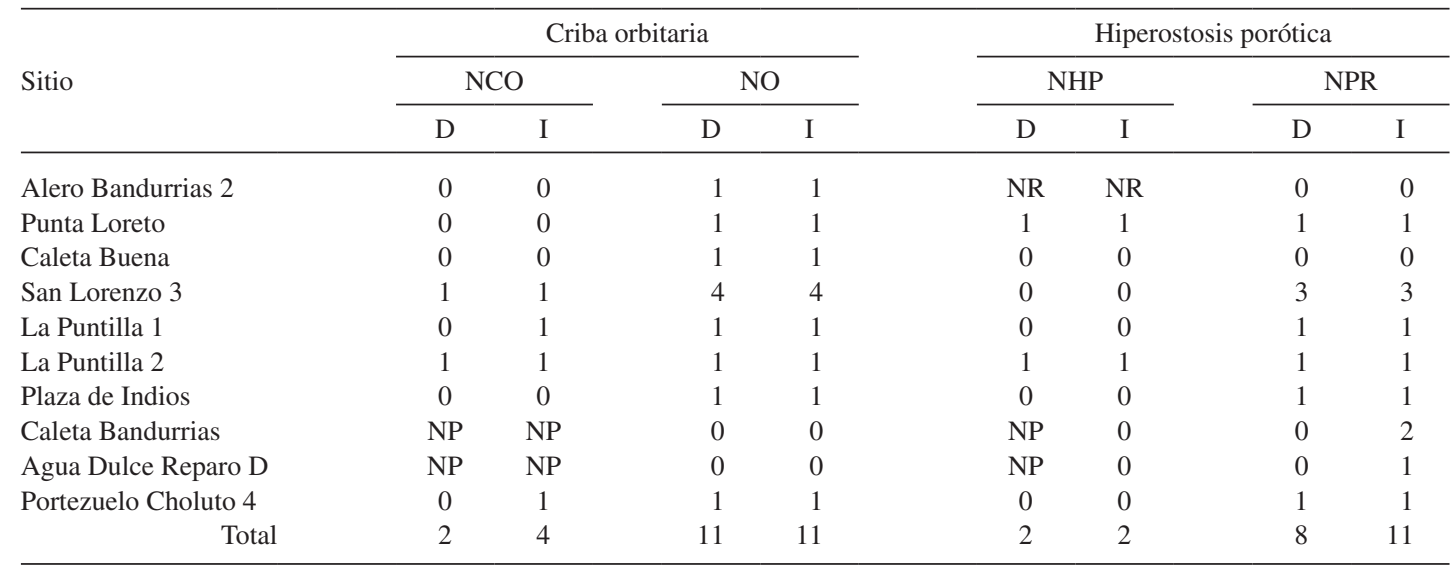

significa que cinco de los 11 individuos presentan enfermedades nutricionales, lo que alcanzaría a un $45,45 \%$ de la muestra (Tabla 3 ).

Además, se realizó un estudio radiológico en tibias con el fin de reconocer líneas de Harris y así determinar si existió algún evento de detención del crecimiento, observándose la presencia de este rasgo en solo una de las 10 tibias analizadas, correspondiente a un individuo del sitio Cachinales 5.

\section{Periostitis y enfermedades infecciosas}

La mayor cantidad de casos de periostitis fueron observados en los miembros inferiores, en donde de un total de 36 individuos posibles de ser analizados, 16 presentaban huellas de periostitis, equivalente al $69,57 \%$ de la muestra analizada, siendo la unidad ósea más afectada la tibia, la que registra esta patología en 19 casos, lo que equivale a $67,85 \%$ (Tabla 4). Estas huellas de periostitis se encontraron en individuos de los sitios Alero Bandurrias 2, San Lorenzo 1, Punta Loreto, Caleta Buena, San Lorenzo 3, Punta Grande, La Puntilla, Los Bronces 5, Cachinales 5, Punta Salitre J14, Agua Dulce Reparo D y 226-5. Por el contrario, en el caso de enfermedades infecciosas, solo pudo observarse un único caso de osteomielitis en una fíbula y en un fémur, ambos correspondientes a uno de los individuos identificados en el sitio Agua Dulce Reparo D.

\section{Enfermedades degenerativas articulares}

\section{Esqueleto apendicular}

Las enfermedades degenerativas estuvieron representadas por casos de osteoartritis (OA), donde se evaluó su presencia a nivel de articulaciones. Considerando la cantidad de articulaciones analizadas, la más afectada corresponde a la cadera, seguida por los hombros y codos, siendo porcentualmente baja en muñecas, rodillas y tobillos (Tablas 5 y 6 ).

\section{Esqueleto axial}

Se analizó un total de 245 vértebras (Tabla 7), pudiendo observarse que la patología degenerativa más frecuente corresponde a la OA, estando presente en el $31,43 \%$ de las vértebras observadas. Ahora bien, dentro de esta frecuencia, se puede observar que las vértebras más afectadas corresponden a las cervicales y torácicas, siendo las vértebras lumbares las que presentan una menor frecuencia de esta afección. En cuanto a la presencia de osteofitos y de nódulos de Schmorl, estos se concentran casi exclusivamente en las vértebras lumbares, siendo su presencia en otras vértebras bastante reducidas.

En el caso del cráneo, se puede decir que la articulación temporo-mandibular (ATM), pudo ser observada en 42 ocasiones reportándose su presencia en 15 casos, lo que equivale a un $35,71 \%$, 
Tabla 4. Presencia de periostitis en el miembro inferior por individuo (PD: Periostitis en lado derecho; PI: Periostitis en lado izquierdo; DDO: Diáfisis derechas observadas; DIO: Diáfisis izquierdas observadas).

Presence of periostitis in the lower limb by individual (PD: Periostitis on the right side; PI: Periostitis on the left side; DDO: Right diaphyses observed; DIO: Left diaphyses observed; NP: Zone or bone not present; NR: Not recordable).

\begin{tabular}{|c|c|c|c|c|c|c|c|c|c|c|c|c|}
\hline \multirow{2}{*}{ Sitio } & \multicolumn{4}{|c|}{ Fémur } & \multicolumn{4}{|c|}{ Tibia } & \multicolumn{4}{|c|}{ Fíbula } \\
\hline & $\mathrm{PD}$ & PI & DDO & DIO & $\mathrm{PD}$ & PI & DDO & $\mathrm{DIO}$ & PD & PI & DDO & DIO \\
\hline Alero Bandurrias 2 & 0 & 0 & 1 & 1 & 1 & 1 & 1 & 1 & 0 & 0 & 1 & 1 \\
\hline San Lorenzo 1 & 1 & 1 & 1 & 1 & NR & NR & 0 & 0 & NR & NR & 0 & 0 \\
\hline Punta Loreto & NP & NR & 0 & 0 & 1 & 1 & 1 & 1 & 0 & NP & 1 & 0 \\
\hline Caleta Buena & 0 & 0 & 1 & 1 & 1 & 0 & 1 & 1 & 0 & 0 & 1 & 1 \\
\hline San Lorenzo 3 & 4 & 3 & 4 & 4 & 4 & 2 & 4 & 2 & 1 & 1 & 1 & 1 \\
\hline Punta Grande & 0 & 1 & 1 & 1 & 1 & 1 & 1 & 1 & 1 & 1 & 1 & 1 \\
\hline La Puntilla 1 & 0 & 1 & 1 & 1 & 1 & 0 & 1 & 1 & 1 & 1 & 1 & 1 \\
\hline La Puntilla 2 & 0 & 1 & 1 & 1 & 1 & 1 & 1 & 1 & 1 & 1 & 1 & 1 \\
\hline Los Bronces 5 & 1 & 1 & 1 & 1 & 1 & NP & 1 & 0 & 0 & NP & 1 & 0 \\
\hline Rescate Riquelme con Carrera & 0 & 0 & 1 & 1 & 0 & 0 & 1 & 1 & NP & NP & 0 & 0 \\
\hline Cachinales 5 & 0 & 1 & 0 & 1 & 0 & NR & 1 & 0 & NP & 0 & 0 & 1 \\
\hline Punta Cachinales 12 & NP & NP & 0 & 0 & NP & NP & 0 & 0 & 0 & NP & 1 & 0 \\
\hline Punta Cañas Norte & 0 & NP & 2 & 0 & 0 & 0 & 1 & 1 & 0 & 0 & 0 & 0 \\
\hline Punta Salitre J4 & 1 & 0 & 1 & 1 & 1 & NP & 1 & 0 & NP & 0 & 0 & 1 \\
\hline Agua Dulce Reparo D & NP & 1 & 0 & 3 & NP & NP & 0 & 0 & NP & 1 & 0 & 1 \\
\hline Portezuelo Choluto 4 & 0 & 0 & 1 & 1 & 0 & 0 & 1 & 1 & NR & 0 & 0 & 1 \\
\hline $226-5$ & NP & NP & 0 & 0 & 1 & NP & 1 & 0 & NP & NP & 0 & 0 \\
\hline 415 Alero 1 & NR & NR & 0 & 0 & 0 & NP & 1 & 0 & NP & NP & 0 & 0 \\
\hline Total & 7 & 10 & 16 & 18 & 13 & 6 & 17 & 11 & 4 & 5 & 9 & 10 \\
\hline
\end{tabular}

registrándose en individuos de los sitios Alero Bandurrias 2, Punta Loreto, San Lorenzo 3, La Puntilla, Plaza Los Indios y Punta Salitre J4.

\section{Patologías inducidas por la subsistencia}

\section{Osteoma auditivo externo}

A partir de la muestra, correspondiente a 13 cráneos susceptibles de ser analizados, se pudo determinar la presencia de este rasgo en tres individuos masculinos de edad adulto joven, provenientes de los sitios Punta Loreto, Plaza Los Indios y La Puntilla, lo que corresponde a un total del 23,08\% de los casos (Tabla 8). Se debe mencionar que en todos ellos la presencia de exostosis fue leve, bilateral y en ningún caso se produjo el bloqueo completo del canal auditivo.

\section{Espondilolisis}

La presencia de espondilolisis pudo detectarse en tres individuos, los cuales corresponden a dos individuos adultos jóvenes de sexo femenino de los sitios Alero Bandurrias 2 y La Puntilla y a un individuo adulto medio de sexo masculino del sitio
Portezuelo Choluto 4. En cuanto a la frecuencia de aparición, se puede decir que en términos de unidades óseas se observaron 59 vértebras lumbares, estando presente únicamente en cuatro, lo que equivale a un 6,78\% de las vértebras (Tabla 7). En términos de individuos, se puede decir que aquellos analizados para esta patología corresponden a 19, por lo que la frecuencia de aparición de la misma sería de un $15,79 \%$.

\section{Retroversión humeral}

La retroversión humeral corresponde a una curvatura de la diáfisis respecto de la cabeza, causado por un estrés muscular en la zona (Kennedy 1989) y por el giro de la articulación (Rhodes 2006). En el caso de la muestra estudiada se puede decir que se pudieron observar 24 tercios proximales de diáfisis de húmeros de ambos lados, observándose en 13 de ellos la torsión de la diáfisis, correspondiente a los sitios Punta Loreto, San Lorenzo 3, Alero Bandurrias 2, Portezuelo Choluto 4, Punta Salitre J4 y La Puntilla 1 (Figura 4). Así, se puede establecer que este rasgo aparece con una frecuencia del $54,17 \%$ (Tabla 8), y si bien no se pudo establecer el sexo de cada uno de los individuos en donde este rasgo 


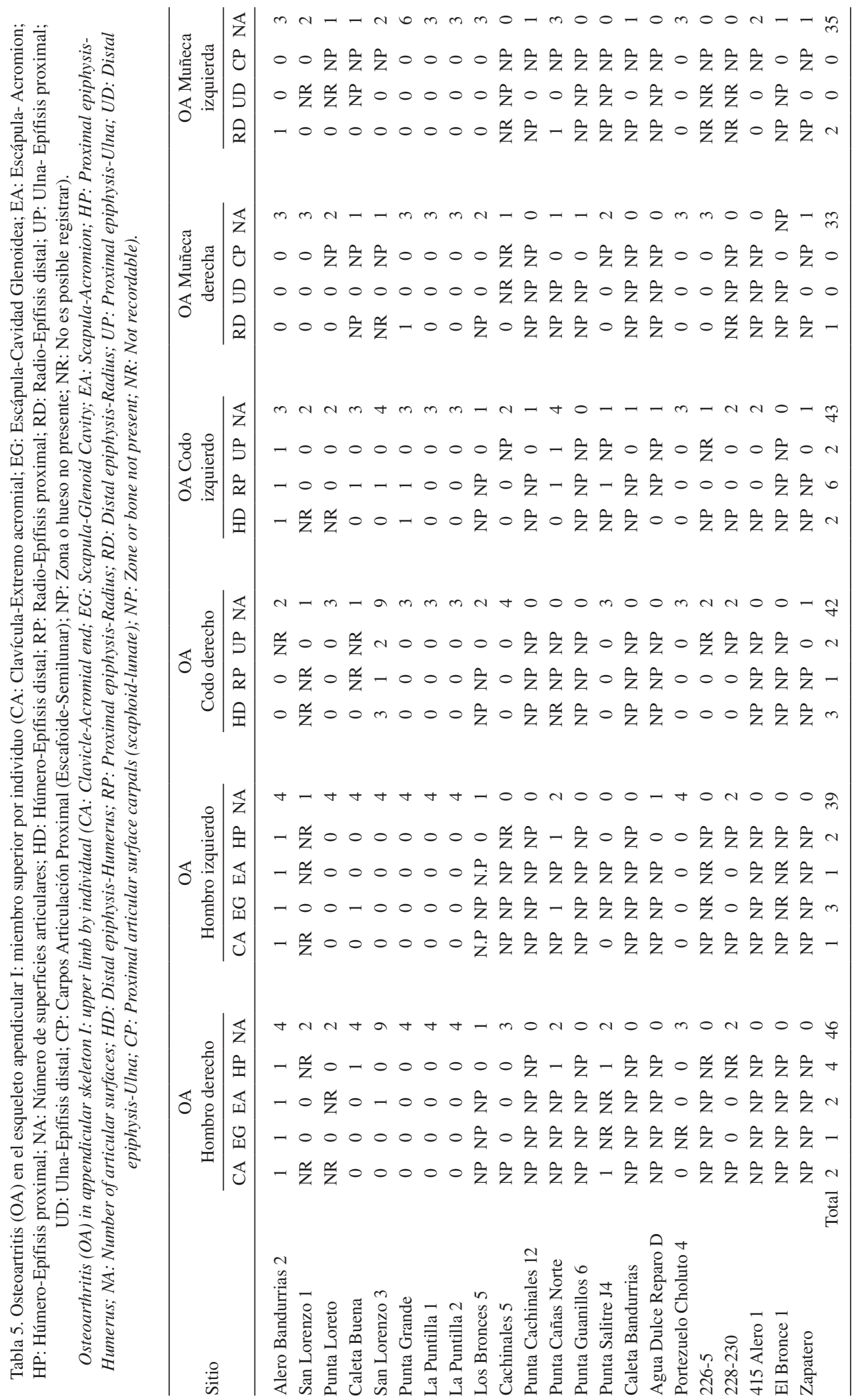




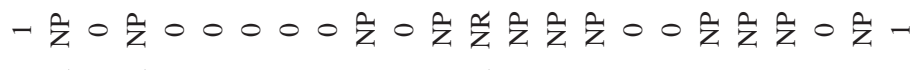

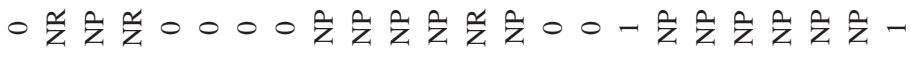

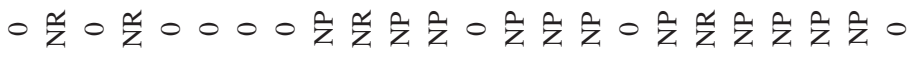

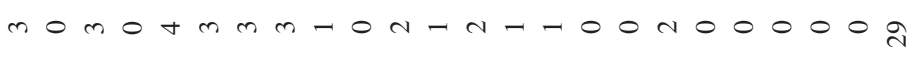

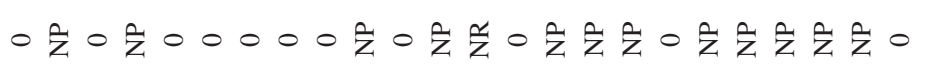

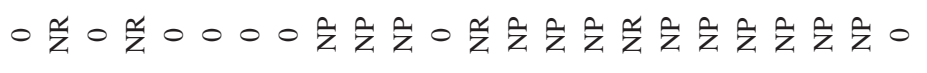

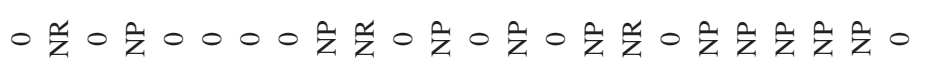
monntmmmnobo-0onnonoo-om

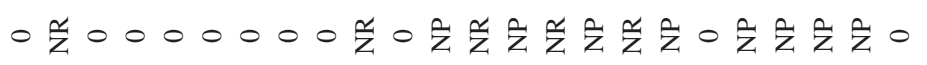

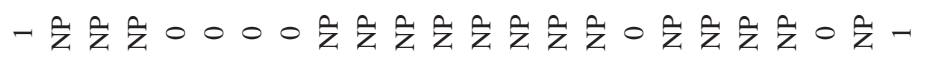

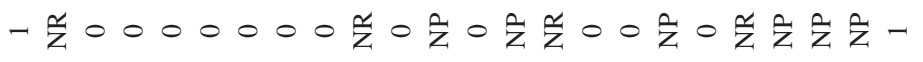
nOnmRmmmo-OmO-O-nOOO-OM

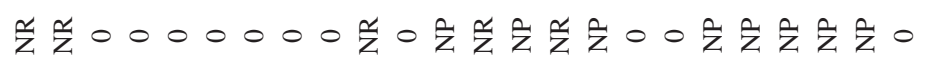

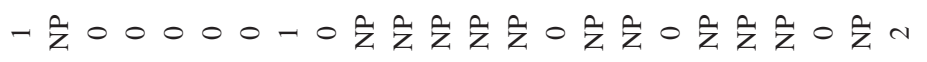

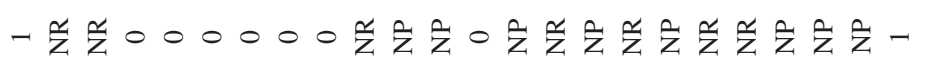
$4 n-7 m 4 n 4-4-0 m 000-04000-8$

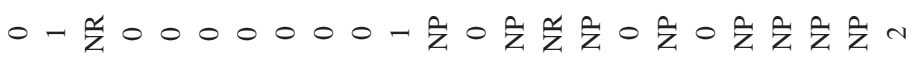

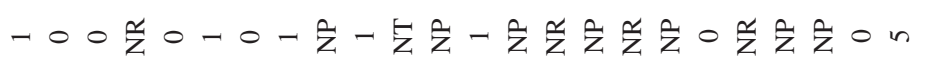

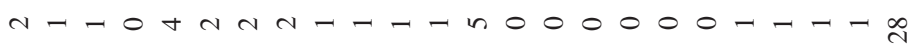

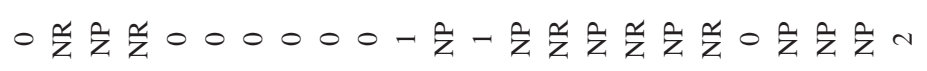

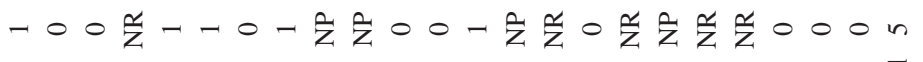

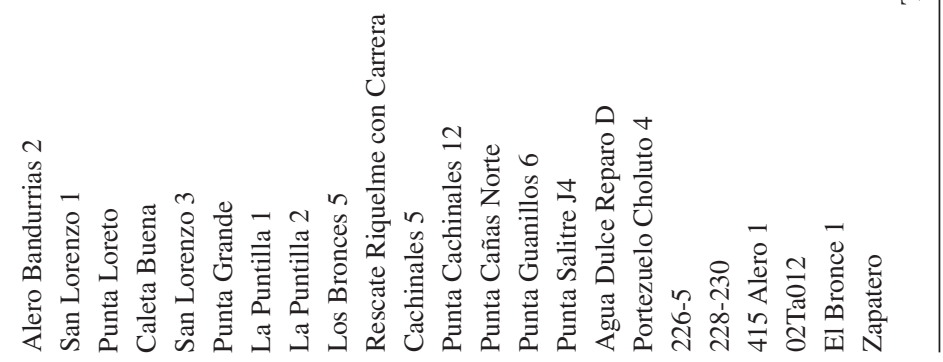



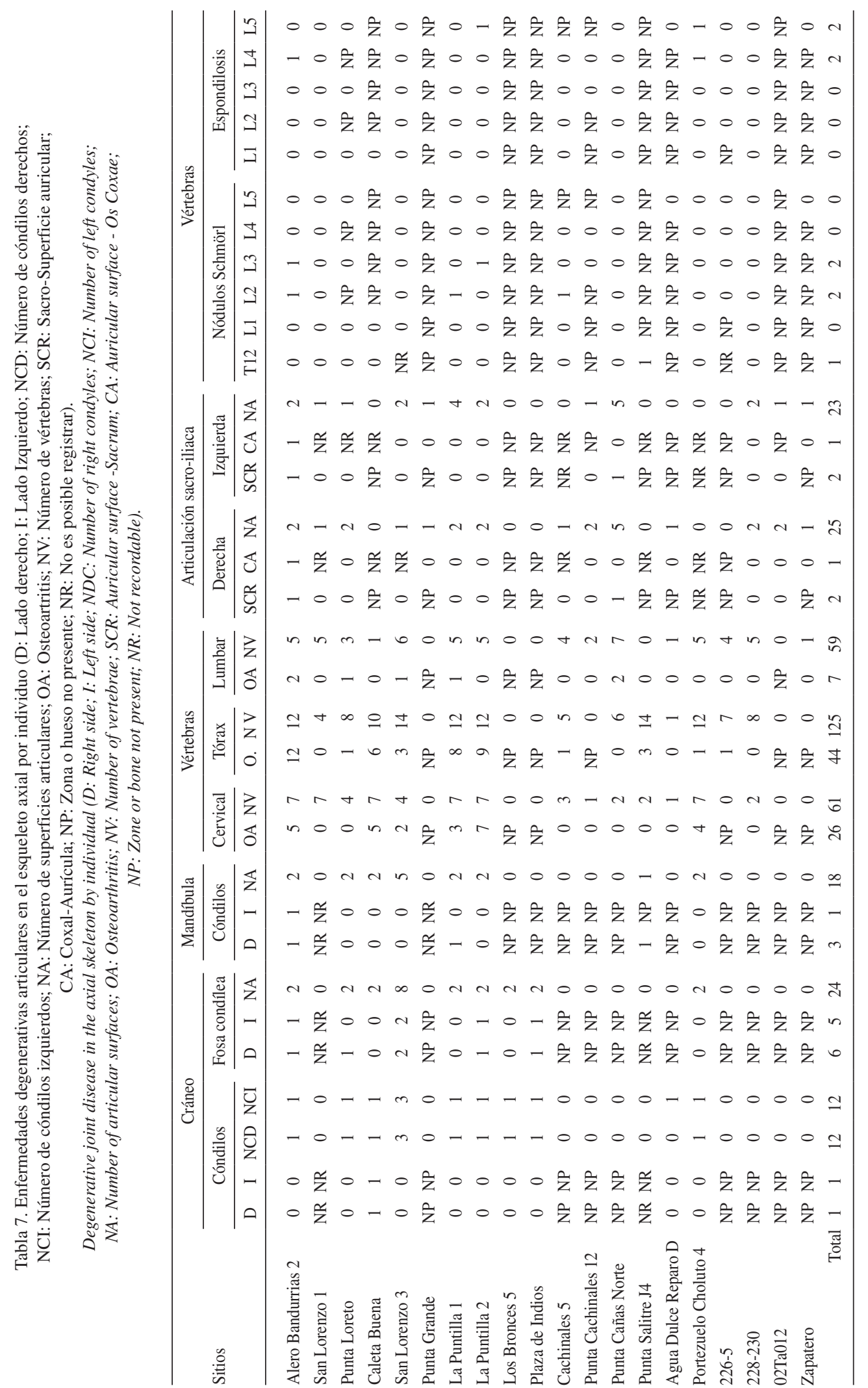


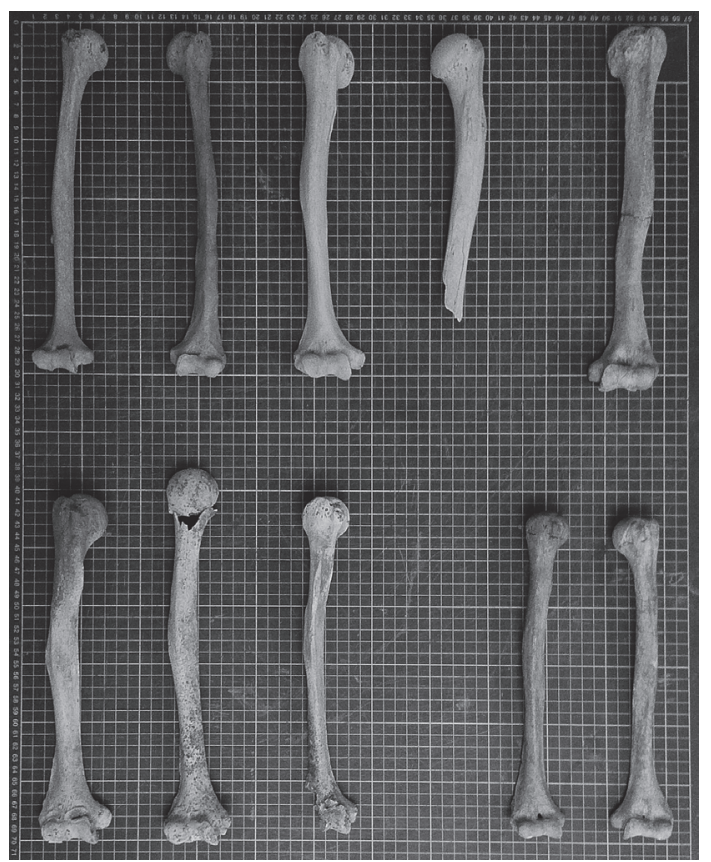

Figura 4. Retroversión humeral identificada en la muestra. Fila superior: Alero Bandurrias 2 (húmeros derecho e izquierdo), Punta Loreto (húmeros derecho e izquierdo) y húmero derecho sin el rasgo. Fila inferior: San Lorenzo 3 (tres húmeros derechos) y húmeros sin el rasgo.

Humeral retroversion identified in the sample. Upper row: Alero Bandurrias 2 (right and left side humeri), Punta Loreto (right and left side humeri) and right humerus without the feature. Lower row: San Lorenzo 3 (three right humeri) and humeri without the feature.

está presente, a partir de los individuos completos analizados se pudo establecer que se encuentra presente tanto en hombres como en mujeres.

\section{Facetas de acuclillamiento}

Las facetas de acuclillamiento constituyen un rasgo posible de observar con bastante frecuencia en la muestra. Así, en 22 tibias observables fue posible evidenciar la presencia de 10 facetas de acuclillamiento, lo que equivale a una prevalencia del $45,45 \%$. Lamentablemente, en este caso no fue posible realizar una determinación de la prevalencia de este rasgo a partir de diferencias de sexo y edad para cada uno de los casos. No obstante lo anterior, nuevamente a partir de esqueletos completos se puede mencionar que este rasgo se encuentra presente tanto en hombres como en mujeres y se expresa en los individuos de los sitios Alero Bandurrias 2, Punta Loreto, San Lorenzo 3, La Puntilla y Punta Cañas Norte (Tabla 8).

\section{Entesofitos en húmeros}

La presencia de entesofitos en las epífisis distales de los húmeros se ha asociado con actividades que involucraban el lanzamiento de objetos arrojadizos, como pueden ser lanzas, arpones, estólicas, entre otros (Kennedy 1989). En nuestra muestra se pudieron observar un total de 24 epífisis distales de húmeros, siendo posible observar en seis casos la presencia de entesofitos, es decir, su prevalencia alcanza un 25\% (Tabla 8), identificándose en los sitios Punta Loreto, Punta Grande, La Puntilla y 226-5. Al igual que en el caso de los rasgos anteriores, se puede decir que no fue posible establecer el sexo y edad en cada individuo que presenta este rasgo, aunque sí es posible establecer que se presentan en adultos de ambos sexos.

\section{Discusión}

Como ya se ha mencionado, la muestra analizada presenta limitaciones por cuanto solo el $52,78 \%$ del total de individuos analizados poseen contextos que permiten asignarlos cronológicamente, mientras que no todos los individuos estudiados se encuentran completos. Pese a ello, los resultados alcanzados son relevantes puesto que constituyen un primer avance hacia la caracterización de los modos de vida de los cazadores-recolectores-pescadores de la costa arreica del norte de Chile desde la bioarqueología. En específico, nuestros resultados nos permiten aproximarnos a las características de la población prehispánica adulta de Taltal y Paposo, dos importantes localidades dentro de la prehistoria regional, pero para las cuales no se habían realizado estudios de este tipo en individuos adultos.

La muestra analizada en el presente trabajo se encuentra dominada por individuos adultos jóvenes, independientemente del sexo que estos posean. Esto puede verse de modo similar en los distribuciones paleodemográficas de las poblaciones costeras con modos de vida cazador-recolector de la costa de Arica y del Norte Semiárido (Arriaza y Standen 2008; Costa-Junqueira, Varela et al. 2000; Quevedo 1998). Lo anterior se encuentra también en relación con las expectativas de vida planteadas para los cazadores-recolectores costeros (Yesner 1980), aunque debido a lo reducido de nuestra muestra no podemos realizar una verdadera apreciación paleodemográfica, con todas sus implicancias sociales (Wood et al. 1992). 


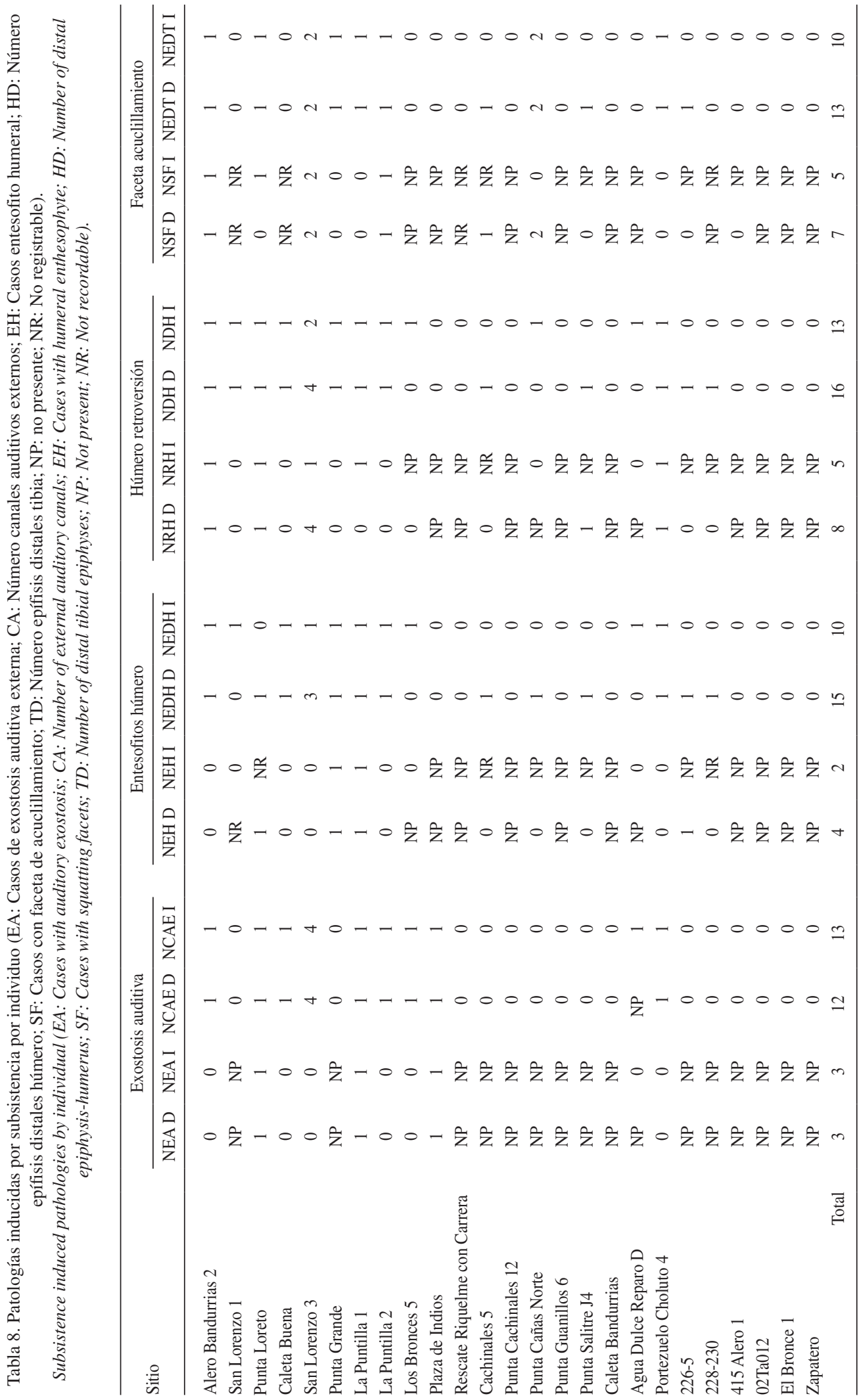


Desde el punto de vista de la estatura se puede decir que existe cierta relación de la muestra estudiada con las poblaciones de la costa de Arica, ya que para los individuos de dicho sector se ha indicado que las mujeres alcanzarían una estatura cercana a los $150 \mathrm{~cm}$, mientras que los hombres alcanzarían los 160 cm (Arriaza 2003; Arriaza y Standen 2008), lo que se asemeja a los rangos obtenidos para los individuos aquí analizados, de 146,58 \pm 4,07 y $158,14 \pm 4,07 \mathrm{~cm}$, respectivamente. Por su parte, se puede observar una diferencia marcada con las estaturas planteadas para los individuos femeninos de las poblaciones del Norte Semiárido, donde las mujeres alcanzarían los $155 \mathrm{~cm}$ y los hombres $160 \mathrm{~cm}$ (Andrade 2008; Quevedo 1998). No es posible por ahora establecer si estas diferencias son o no significativas desde un punto de vista estadístico, debido al escaso número muestral.

Más informativos resultaron los datos sobre paleopatologías e indicadores de estrés ocupacional. Entre estos, debe destacarse la alta presencia de enfermedades metabólicas, las cuales corresponden a criba orbitaria e hiperostosis porótica. Ambas condiciones paleopatológicas están altamente relacionadas con situaciones de estrés ocasionadas por la privación de nutrientes y anemia como resultado de factores dietarios y no dietarios, como la falta de ingesta de comidas ricas en hierro, parasitarismo, diarreas infantiles (Larsen 1995:199; Rothschild 2000; Stuart-Macadam 1989, 2005) y por transmisión genética (Sisson y Lund 1958; Walker 1985). Otras investigaciones arqueológicas han sugerido que las manifestaciones de las enfermedades nutricionales poseen una mayor prevalencia en agricultores sedentarios que en cazadores-recolectores, debido a que los primeros tendrían una mayor exposición a focos de contaminación y a que el consumo de recursos vegetales es la base de su dieta (Armelagos 1990:132; Cohen y Armelagos 1984). No obstante, en nuestra muestra la presencia de estas condiciones paleopatológicas alcanza una frecuencia de un $45,45 \%$, lo que contrasta con el $24 \%$ reportado para las poblaciones cazadoras-recolectoras de Arica (Arriaza 2003:127) y el 19,28\% observado para los grupos Arcaicos de la costa del Norte Semiárido (Andrade 2008). Esta situación podría explicarse en términos de un alto consumo de peces y mamíferos marinos que contengan parásitos (Reinhard y Urban 2003; Santos y de Faro 2005), privación de alimentos, consumo de agua contaminada, transmisión genética y situaciones ambientales que propician la aparición de esta enfermedad, como se ha interpretado para distintas poblaciones cazadoras-recolectoras con una dieta basada en el consumo de recursos marinos (Arriaza 2003:127; Cybulski 1977; Keenleyside 1998; Storey et al. 2002; Walker 1986; White et al. 2006).

También se exploró la presencia de líneas de Harris en la muestra estudiada. Esta expresión patológica corresponde a una respuesta a momentos de estrés fisiológico cuando el crecimiento de las piezas óseas fue comprometido, por lo que su presencia solo puede darse cuando el individuo está en proceso de crecimiento, es decir, durante la infancia, pudiendo relacionarse con enfermedades nutricionales o con otras condiciones paleopatológicas presentes en la infancia (Roberts y Manchester 2005:240). La virtual ausencia de esta condición en nuestra muestra contrasta con la situación reportada para las poblaciones del Arcaico Tardío de Arica (sitio Morro 1), donde las líneas de Harris presentan en una frecuencia de 84,6\% (Arriaza et al. 1984), situación que se repite también en el Norte Semiárido donde aparecen con una frecuencia cercana al $60 \%$ (Quevedo et al. 2000). Tal como plantean Hummert y Van Gerven (1985), es posible que las líneas de Harris desaparezcan debido a remodelaciones óseas que se producen con la edad. Por lo tanto, las diferencias observadas pueden deberse a que nuestra muestra solo consta de individuos adultos, en los cuales la presencia de esta patología podría estar oscurecida.

En el caso de las huellas de periostitis, se puede decir que estas corresponden a una de las más recurrentes observadas en la muestra (cercana al $70 \%$ ), principalmente en el caso de los miembros inferiores. Ahora bien, la periostitis presenta una gran dificultad en cuanto a su diagnóstico, ya que no corresponde a una enfermedad en sí, sino a una reacción del periostio, principalmente de los huesos largos, frente a ciertos estímulos, entre los que se cuentan agentes infecciosos no específicos, como también a eventos traumáticos constantes en las zonas afectadas, postulándose que los grupos cazadores-recolectores tendrían menor incidencia de periostitis que los grupos productores de alimentos (Armelagos 1990; Cohen y Armelagos 1984; Larsen 1995, 1997; Roberts 2000; Weston 2008).

En el caso de los individuos recuperados de la zona de Taltal y Paposo se puede decir que si estos hubiesen estado expuestos a agentes infecciosos, la presencia de periostitis se vería también en otros 
huesos largos y también con manifestaciones distintas. El hecho de que solo existe un único caso de osteomielitis, la cual corresponde a una respuesta producto de una enfermedad infecciosa con un agente causante desconocido (Larsen 1995), refrenda que la periostitis observada debió ser causada por eventos traumáticos que afectaron los miembros inferiores, relacionados ya sea con la marcha sobre terrenos escarpados, la exposición a las frías aguas del océano Pacífico y/o las actividades extractivas en general. En el caso de las poblaciones de Arica, Costa-Junqueira et al. (2000b) reportan un $18,18 \%$ de individuos con periostitis y un $21,69 \%$ de osteomielitis, mientras que para la costa del norte semiárido Quevedo et al. (2000) indican la presencia de un $14 \%$ de individuos con periostitis, $28 \%$ de individuos con osteomielitis, así como un $26 \%$ de individuos con ambas patologías. La mayor prevalencia de esta condición en los individuos encontrados en Taltal y Paposo podría obedecer al sistema de asentamiento local que implicó una alta movilidad residencial y constantes desplazamientos a lo largo del litoral y entre este y el interior en busca de materias primas.

Las enfermedades degenerativas identificadas en la muestra podrían obedecer a una serie de actividades realizadas por los individuos. Así, la presencia de osteoartritis en la ATM se puede explicar como el producto de una fuerte presión masticatoria realizada para trabajar cueros o fibras con los dientes (Malgosa 2003). Por su parte, la presencia de OA en los hombros se puede relacionar con la elevación intensa de los brazos, lo que se asocia a la extensión y flexión del brazo bajo gran presión, lo que en el contexto de la zona costera de donde provienen los restos analizados, podría asociarse tanto con el lanzamiento de elementos arrojadizos para la caza, como lanzas, estólicas y arpones, como con la acción de remar (Malgosa 2003). De todas formas se debe mencionar que estas actividades no son excluyentes y es probable que todas ellas estén evidenciadas en las unidades óseas.

Desde un punto de vista comparativo, según datos compilados por Arriaza y Standen (2008:76) para Arica, llaman la atención algunas importantes diferencias, como la mayor incidencia de OA en las articulaciones de nuestra muestra, a excepción de la rodilla, la cual es más alta en Arica. En ese mismo sentido, llama la atención la nula presencia de OA en las caderas, la cual corresponde a la articulación más afectada en la muestra de Taltal-Paposo. En el caso del esqueleto axial también se observan diferencias, presentando la muestra estudiada mayor prevalencia de OA en el caso de las vértebras cervicales, vértebras torácicas y ATM, pero una menor en el caso de las vértebras lumbares.

En el caso de la muestra del Norte Semiárido, la información entregada por Quevedo (2000) y Quevedo et al. (2003) indica mayores frecuencias de aparición de OA en tobillo, muñeca y codo, mientras que la muestra de Taltal-Paposo presenta mayor aparición de OA hombro y cadera, la cual en la muestra del Norte Semiárido es la con menor frecuencia de afección.

Sobre el caso específico de la OA en la cadera se debe mencionar que la causa de esta lesión se produce por actividades relacionadas con la estabilización del peso sobre la articulación durante la marcha y a la tensión que se produce en los músculos de la zona (Bombellini 1976; Knüsel 2000). Lo anterior tendría relación con la adopción de posturas forzadas, como el acuclillamiento, las marchas por terrenos complicados y la carga constante de peso extracorporal.

En términos generales, se puede decir que la muestra de Taltal-Paposo supera ampliamente en términos de presencia de OA a aquellas de Arica y del Norte Semiárido. Tal vez la explicación de estas diferencias sea resultado de una diversificación entre las actividades realizadas por los individuos de la costa arreica, en comparación con aquellas realizadas por los habitantes arcaicos de la costa de Arica y del Norte Semiárido. No obstante, el conjunto artefactual para la extracción y procesamiento de recursos litorales asociado cronológicamente a nuestra muestra fue muy semejante al reportado para el Arcaico Tardío de los Valles Occidentales, por lo que cabe esperar que las actividades extractivas hayan sido también muy parecidas. En este escenario, quizás las actividades llevadas a cabo por los individuos de nuestra muestra serían más severas y/o constantes, que aquellas llevadas a cabo por los miembros de las otras poblaciones. Vale decir, la mayor frecuencia de actividades causante de patologías degenerativas en los individuos de la costa de Taltal y Paposo en relación con los de Arica parece indicar condiciones de vida más exigentes, quizás como resultado de la escasez de agua o, más probablemente, los sistemas de asentamiento más móviles en comparación con Valles Occidentales, los que implicaron más intensidad y recurrencia en marchas por terrenos escabrosos. A lo anterior 
también podríamos sumarle una eventual mayor dependencia de las poblaciones de Taltal y Paposo en la caza del lobo marino y el uso de embarcaciones, quizás ante la ausencia de zonas de eficiencia de desembocadura de ríos o acceso a valles interiores.

Desde el punto de vista de las enfermedades degenerativas observadas en la columna vertebral, las más recurrentes corresponden a OA y osteofitos, estos últimos principalmente en las vértebras lumbares. La presencia de estas patologías puede relacionarse con una carga de peso constante en las distintas zonas de la espalda, así como también con movimientos que involucran la flexión y extensión del tronco, como también su estabilización (Larsen 1997:163-164). Lamentablemente, la falta de datos detallados a nivel de vértebras afectadas para los grupos arcaicos costeros de Arica y del Norte Semiárido impiden por ahora realizar una comparación regional más acabada al respecto. Pero dados los ya comentados resultados comparativos en términos de osteoartritis en las articulaciones, creemos que la situación en este caso sería similar a lo anteriormente planteado.

Respecto de las enfermedades asociadas a la subsistencia, tenemos la presencia de exostosis auditiva externa en la muestra estudiada, la cual se asocia a tres individuos del sexo masculino. Este rasgo se ha vinculado a la pesca submarina (Kennedy 1989; Standen et al. 1995, 1997), siendo asociada en los grupos arcaicos costeros de Arica y del Norte Semiárido principalmente a los individuos adultos de sexo masculino, aun cuando en esta zonas se menciona también la aparición ocasional de osteoma en mujeres (Arriaza 2003; Quevedo 2000; Standen et al. 1995, 1997).

Por su parte, la presencia de espondilólisis en nuestra muestra presenta algunas diferencias respecto de los individuos de otras poblaciones. Esta patología se ha relacionado con movimientos constantes, vigorosos y repetidos de la parte baja de la espalda, como aquellos relacionados con el levantamiento de peso y el remo, entre otros (Roberts y Manchester 2005:106). En el caso de poblaciones costeras con un modo de subsistencia basada en la caza y recolección, la presencia de espondilólisis ha sido registrada en Arica, siendo observada únicamente en individuos de sexo masculino, en una frecuencia cercana al $20 \%$, lo que se ha interpretado como una diferenciación de actividades en términos del sexo (Arriaza 2003:131-132). Esto señala una clara diferencia respecto de la muestra aquí analizada, ya que en este caso, la presencia de espondilólisis se registró en dos individuos adultos jóvenes femeninos y en un individuo adulto medio masculino. En este sentido, podría plantearse de manera preliminar que las comunidades de cazadores-recolectores de la zona de Taltal-Paposo tendrían menor diferencia de género en términos de las actividades desarrolladas, lo que implicaría asimismo que las mujeres aquí analizadas realizaban un espectro más amplio de actividades que las mujeres de la costa de Arica, lo que se condice también con la alta frecuencia de enfermedades degenerativas en los individuos femeninos de Taltal-Paposo.

La presencia de facetas de acuclillamiento se puede ver en individuos de ambos sexos y muchas veces de forma bilateral, es decir, en ambas tibias. Este rasgo se debe a la mantención constante y prolongada de una postura acuclillada, involucrando la flexión extrema de las articulaciones de la cadera, rodilla y tobillo (Larsen 1997:185). No obstante, existen antecedentes que vinculan esta patología también con una gran demanda muscular en los miembros inferiores, como resultado por ejemplo de caminatas constantes y/o prolongadas (Trinkaus 1975), y también el tránsito por terrenos escarpados (Angel 1971). En el caso de los individuos aquí analizados, debido a las condiciones geográficas del sector de los hallazgos y el patrón de asentamiento altamente móvil de la época, no se puede descartar que sea una combinación de ambos factores. La presencia de facetas de acuclillamiento han sido reportadas por Quevedo (2000) como de frecuencia baja para la población de Punta Teatinos y aunque se indica que están más presentes en hombres, no se entregan más detalles. Una situación similar ocurre la muestra del Arcaico de Arica, donde Standen et al. (1984) indican que el rasgo se encuentra únicamente en mujeres, no entregando más detalles en cuanto a frecuencias.

Junto con lo anterior, se pudo observar en un importante porcentaje de casos la presencia de retroversión humeral, en individuos de ambos sexos (Figura 4). Esta torsión se ha asociado a movimientos constantes relacionados con actividades sistemáticas y recurrentes que involucran a los brazos, donde se produce una fuerte flexión y extensión de las articulaciones del brazo (Malgosa 2003:229), afines a la tracción de objetos pesados con ambas extremidades (Constantinescu 2001). Además, existen autores que han relacionado la presencia de estas torsiones a actividades relacionadas con la boga y la navegación (Jurmain y Kilgore 1995; 
Molnar 2006). En el caso de los individuos aquí analizados y en coherencia con otros bioindicadores disponibles desde el Arcaico Medio (Olguín et al. 2014), es altamente probable que esta condición patológica sea resultado del uso de embarcaciones a remo, en cuyo caso esta actividad estaría siendo llevada a cabo tanto por hombres como por mujeres, lo que nuevamente muestra una escasa diferencia de actividades a nivel de género en la comunidad local, aunque lo anterior debe ser complementado con otros estudios de registro de entesis. Sobre este rasgo en específico no encontramos evidencias en las poblaciones que se han ocupado para las comparaciones, siendo el dato más cercano el reportado por Quevedo (2000) que relaciona las lesiones de osteartritis del miembro superior con actividades ligadas a la navegación.

Finalmente, se pudo detectar la presencia de entesofitos en un $25 \%$ de húmeros observados. Este rasgo corresponde a una exostosis en la epífisis distal provocada por la hiperactividad de diversos músculos del brazo, antebrazo y de la mano (Malgosa 2003:228). En estudios de poblaciones prehistóricas se ha asociado la presencia de esta condición paleopatológica a actividades relacionadas con el lanzamiento de elementos o artefactos arrojadizos (Kennedy 1989), como pueden ser lanzas, estólicas o arpones (Molnar 2006), actividades que en nuestra área de estudio estarían siendo realizadas por individuos de ambos sexos. Nuevamente, si bien en la bibliografía de las muestras utilizadas como referente (Arriaza 2003; Quevedo 2000;) se habla de que los individuos habrían realizado lanzamiento de elementos arrojadizos, no encontramos referencias directas sobre la prevalencia de esta reacción patológica.

A manera de síntesis, es relevante destacar que desde un punto de vista cronológico, la muestra estudiada no exhibe diferencias significativas. Vale decir, la mayor parte de los rasgos observados en Taltal-Paposo se encuentran representados en el Arcaico Tardío, Alfarero Temprano, Medio y Tardío, así como en la muestra de individuos no cronologizados. Excepciones a lo anterior lo constituye la ausencia de exostosis auditiva en el Arcaico Tardío, de entesofitos en el húmero en el Alfarero Temprano, de nódulos de Schmorl en el Alfarero Tardío y de espondilosis en el Arcaico Tardío y el Alfarero Temprano. No obstante, dada la baja frecuencia total de estos marcadores y las dificultades para identificar estas patologías en los individuos incompletos, lo más probable es que su ausencia durante ciertos períodos se deba a los problemas inherentes a la muestra estudiada y que no representen condiciones reales durante la prehistoria. Lo anterior parece refrendarse en el caso de la exostosis auditiva, ya que esta es una patología frecuente en poblaciones arcaicas costeras, tanto de Arica como del Norte Semiárido e incluso de la costa arreica (Ardiles et al. 2011; Arias y Herrera 2012), por lo que probablemente sí se presentó en Taltal-Paposo en el Arcaico Tardío.

Por otro lado, puede señalarse que, tomada en su conjunto (sin discriminación cronológica) y en comparación con individuos cazadores-recolectores costeros de áreas vecinas, la muestra acá estudiada presenta rasgos comunes con Valles Occidentales y el Norte Semiárido, pero también muestra ciertas particularidades que merecen ser destacadas. En primer lugar, llama la atención la ausencia de indicadores de violencia interpersonal en TaltalPaposo, lo que contrasta notablemente con el $30 \%$ que alcanza esta condición entre la población masculina Chinchorro de Arica (Standen y Arriaza 2000; véase también Costa-Junqueira et al. 2000b). Los patrones de alta movilidad residencial que caracterizaron a la prehistoria de nuestra área de estudio con posterioridad al $4.500 \mathrm{cal}$ a.p. pudieron haber incidido en menores índices de violencia interpersonal en comparación con una población como la Chinchorro que experimentó un aumento demográfico y un sistema de asentamiento semisedentario con potenciales conflictos por el control de los recursos (Marquet et al. 2012; Santoro et al. 2012). Por otro lado, también parecen ser menores las enfermedades infecciosas en nuestra muestra de estudio, lo cual también sería esperable en contextos de alta movilidad residencial y poca permanencia de las ocupaciones en los campamentos base.

Adicionalmente, nuestra muestra evidencia una mayor proporción de criba orbitaria e hiperostosis porótica. Es difícil atribuir estas patologías a estrés ambiental, ya que los recursos disponibles en valles occidentales y en la costa arreica habrían sido muy similares, a excepción por supuesto del agua dulce. En este sentido, un ambiente de arreísmo como el que caracterizó a la costa entre Pisagua y Chañaral (a excepción de caleta Huelén) podría haber propiciado almacenamiento o el consumo de agua estancada, la que puede presentar alto contenido mineral, todo lo cual pudo contribuir a producir enfermedades metabólicas. No obstante, el hecho de que en Taltal-Paposo esta condición 
paleopatológica sea aún más frecuente que en las poblaciones de Chacaya en Antofagasta (Ardiles et al. 2011) y de Cáñamo 3 en Iquique (Arias y Herrera 2012), ambas correspondientes al Arcaico Tardío y el Alfarero Temprano de la costa arreica respectivamente, nos sugiere que la escasez de agua de la costa arreica no es suficiente para explicar la frecuencia de criba orbitaria e hiperostosis porótica en nuestra muestra. Es posible hipotetizar que la variable causante haya sido prácticas alimentarias diferenciales, las cuales en Taltal-Paposo habrían propiciado mayormente el parasitarismo. Futuras investigaciones deberán confirmar esta posibilidad.

Otra diferencia interesante entre la población de Taltal y Paposo respecto del Arcaico de Arica, es la menor diferenciación de actividades en términos de género, siendo la única excepción el caso de la exostosis auditiva. El hecho de que las comunidades Chinchorro de Arica hayan presentado mayores índices de diferenciación de actividades por sexo podría reflejar una sociedad más compleja, con alta demografía y patrón de asentamiento semisedentario, en contraste con comunidades presumiblemente más pequeñas y más móviles en Taltal y Paposo entre el 4.500 y el 700 cal a.p., lo que habría inhibido el desarrollo de tales diferencias.

No obstante lo anterior, cabe destacar que los individuos analizados en nuestra área de estudio sí exhiben ciertas diferencias internas en términos de condiciones paleopatológicas e indicadores de estrés ocupacional, aun cuando estas no son significativas en cuanto al sexo de los individuos. Ya hemos señalado que tales diferencias tampoco tienen significancia cronológica dentro de la muestra estudiada, pero en el futuro será interesante precisar si responden o no a una especialización de ciertos individuos en tareas específicas dentro de la economía de subsistencia de estas poblaciones. Un ejemplo de lo anterior podría ser la exostosis auditiva, ya que en todos los períodos existen individuos masculinos que no presentan esta patología. Esto podría sugerir que las actividades de buceo (Standen et al. 1995) no fueron practicadas por todos los individuos masculinos del grupo, tal como sucede en otras zonas. Lo mismo cabe señalar respecto de las facetas de acuclillamiento y algunas enfermedades degenerativas, las cuales no están presentes en todos los individuos de la muestra y en consecuencia podrían sugerir la especialización de ciertos individuos -independiente de su sexo-en labores de recolección de orilla, la caza de lobos marinos o la navegación, entre otras. Este tipo de diferencias al interior de la población de cazadoresrecolectores-pescadores del área de Taltal y Paposo nos parecen significativas a la luz de los planteamientos de Castelleti (2007) y Salazar (2013), quienes observan desde el Arcaico Tardío la profusión de sitios de tareas específicas que denotarían un sistema de asentamiento de carácter más logístico y con mayor diversificación de emplazamientos y presumiblemente de actividades. No existen antecedentes por ahora para asumir que tales diferencias en las actividades tuvieron implicancias sociales.

\section{Conclusiones}

El estudio paleopatológico y las actividades inferidas a partir del análisis de la muestra permiten postular que los individuos estudiados corresponden a miembros de una población con un modo de subsistencia tradicional ligado a la caza, recolección y pesca de recursos litorales. Lo anterior se confirma adicionalmente al constatar tanto el registro arqueológico asociado (artefactos y ecofactos) como recientes resultados de análisis de isótopos estables actualmente en proceso de estudio, obtenidos de los sitios Caleta Buena, Punta Cañas Norte, Punta Loreto, San Lorenzo 1, San Lorenzo 3 y Zapatero. Si bien estos individuos abarcan una extensión cronológica de casi 4000 años, los indicadores isotópicos son virtualmente idénticos en todos ellos.

En nuestra área de estudio no solo habría una continuidad en la dieta desde el Arcaico Tardío hasta el Alfarero Tardío, sino que las principales actividades realizadas por los individuos se mantendrían relativamente estables a lo largo del tiempo. Estas actividades corresponden a aquellas ligadas con la carga y levantamiento de peso, lo que se refleja en la gran cantidad de enfermedades degenerativas presentes en la columna vertebral; la presencia en los miembros superiores de huellas relacionadas con el uso de remo y/o empleo sistemático de objetos arrojadizos, lo que debiera asociarse con la constante presencia de restos óseos de otáridos en el registro arqueofaunístico (Salazar 2013) o bien con la caza de grandes especies epipelágicas como la albacora, el marlín o los tiburones constatada desde el Arcaico Medio en el área de estudio (Olguín et al. 2014). Además de lo anterior, fue posible establecer que las patologías observadas en los miembros inferiores dan cuenta de un uso constante de estas extremidades, las que probablemente estén ligadas a marchas permanentes y/o prolongadas, a la mantención de 
posturas forzadas de acuclillamiento por un largo periodo en actividades de recolección de recursos marinos, e incluso a una combinación de ambas. Lo anterior se relaciona también con la presencia de recursos de orilla en los sitios de estos períodos, así como con el patrón de asentamiento local que evidencia alta movilidad residencial a contar del Arcaico Tardío (Salazar et al. 2009). La alta incidencia de enfermedades metabólicas en la muestra analizada parece vincularse a prácticas alimentarias. Trabajos anteriores han demostrado que el parásito Diphyllobotrium pacificum se transmite por el consumo de pescados y mamíferos marinos crudos o poco cocidos, mientras que este parásito ha sido reportado en diversos contextos prehistóricos tanto de los valles de Arica como de Tarapacá (Ferreira et al. 1984; Núñez y Hall 1982; Reinhard y Urban 2003). Dada la alta movilidad residencial posterior al Arcaico Tardío, el hacinamiento no habría sido condición de transmisión de este parásito, sino más bien el reiterado consumo de los alimentos que los alojan. Si bien todas estas actividades se encuentran presentes en la muestra estudiada, demostrando la continuidad histórica del modo de vida, no todos los individuos presentan el mismo cuadro paleopatológi$\mathrm{co}$, por lo que es posible que haya habido una cierta especialización en las actividades de procuramiento y preparación de los bienes de subsistencia entre los individuos que conformaron las comunidades de cazadores-recolectores y pescadores de Taltal y Paposo entre el 4.500 y el 700 cal a.p.
Por último, debemos señalar que pese a que la muestra analizada presenta limitaciones en cuanto a su composición y contexto, creemos que la consistencia en los resultados obtenidos demuestra que se trata de una población representativa de los habitantes prehispánicos de la zona. En este sentido, el presente trabajo pretende ser una primera contribución al conocimiento de su modo de vida y subsistencia desde el registro bioarqueológico, siendo posible respaldar y complementar las inferencias que se han realizado anteriormente desde la materialidad arqueológica.

Agradecimientos: El presente trabajo es resultado de los Proyectos Fondecyt 1080666 y 1110196. Agradecemos a todos los colegas, estudiantes y amigos que conforman el equipo de terreno y laboratorio de nuestros proyectos por su constante apoyo y compromiso. Los autores también agradecen a los señores Rodolfo Contreras e Ivo Kuzmanic de los Museos de Taltal y Antofagasta, respectivamente, por el acceso y disponibilidad para revisar los materiales depositados en sus instituciones. Hacemos un especial reconocimiento a Paulina Chávez por la elaboración de la Figura 1 y a César Borie por la elaboración de la Figura 2. Por último, quisiéramos reconocer el aporte del comité editorial de la revista y de los evaluadores anónimos para la mejoría del artículo. No obstante todo lo anterior, los errores del texto son obviamente de nuestra exclusiva responsabilidad.

\section{Referencias Citadas}

Adams, B.J. y L.W. Koningsberg 2004. Estimation of the most likely number of individuals from commingled human skeletal remains. American Journal of Physical Anthropology 152:138-151.

- - - 2008. How many people? Determining the number of individuals represented by commingled human remains. En Recovery, Analysis, and Identifaction of Commingled Human Remains, editado por B.J. Adams y J.E. Byrd, pp. 241-256. Humana Press, New York.

Alfonso, M., V. Standen y V. Castro 2007. The adoption of agriculture among northern Chile populations in the Azapa Valley, 9000-1000 BP. En Ancient Health: Skeltal Indicators of Agricultural and Economics Intesification, editado por M. Cohen y G. Crane-Kramer, pp. 113-129. University of Florida Press, Gainesville.

Andrade, P. 2008. Lifestyle reconstruction of an Early Pottery Period Population from the Choapa River Basin, IV Region, Chile: A Comparative Study from the Archaeological Site MLP PTF 13. A Dissertation Submitted in Partial Fulfillment of the Requirements for MA Osteoarchaeology by Taught Course, Department of Archaeology, University of Southampton, Southampon.

Andrade, P. y D. Salazar 2011. Revisitando Morro Colorado: comparaciones y propuestas preliminares en torno a un conchal arcaico en las costas de Taltal. Taltalia 4:63-83.

Angel, J.L. 1971. Lerna: a Preclassical Site in the Argolid. Results of Excavations Conducted by the American School of Classical Studies at Athens. Volume II: The people. American School of Classical Studies at Athens, Princeton and Smithsonian Institution Press of Washington, Washington.

Ardiles, H.; B. Ballester y A. Clarot 2011. Elección de dieta en poblaciones pasadas de la costa de la II Región: una mirada multidisciplinaria. En Informes del Fondo de Apoyo a la Investigación Patrimonial, pp. 83-110. Dirección de Bibliotecas, Archivos y Museos, Santiago.

Arias, M. y M.J. Herrera 2012.Caracterización del modo de vida, salud y dieta de las poblaciones de los cementerios CaseronesTarapacá 40 y Cáñamo 3 (Período Formativo, Región de 
Tarapacá). Actas del XVIII Congreso Nacional de Arqueología Chilena, pp. 45-54, Arica.

Armelagos, G.J. 1990. Health and Disease in Prehistoric Populations in Transition. En Disease in Populations in Transition. Anthropological and Epidemiological Perspectives, editado por A.C. Swedlund y G.J. Armelagos, pp. 127-144. Bergin \& Garvey, New York.

Arriaza, B. 1993. Seronegative spondyloarthropathies and diffuse idiopathic skeletal hyperostosis in ancient northern Chile. American Journal of Physical Anthropology 91:263-278.

- - - 2003. Cultura Chinchorro. Las Momias Más Antiguas del Mundo. Editorial Universitaria, Santiago.

Arriaza, B., M. Allison y V. Standen 1984. Líneas de Harris en una población Arcaica Tardía del extremo norte de Chile: Morro-1. Chungara Revista de Antropología Chilena 13:187-191.

Arriaza, B., L.C. Cartmell, C. Moragas, A.G. Nerlich, W. Salo, M. Madden y C. Aufderheide 2008. The bioarchaeological value of human mummies without provenience. Chungara Revista de Antropología Chilena 40:55-65.

Arriaza, B., W. Salo, A. Aufderheide y T. Holcomb 1995. PreColumbian tuberculosis in Northern Chile: Molecular and skeletal evidence. American Journal of Physical Anthropology 98:37-45.

Arriaza, B. y V. Standen 2008. Bioarqueología. Historia Biocultural de los Antiguos Pobladores del Extremo Norte de Chile. Editorial Universitaria, Santiago.

Aufderheide, A., S. Aturaliya y G. Focacci 2002. Pulmonary disease in a sample of mummies from the Az-75 cemetery in Northern Chile's Azapa Valley. Chungara Revista de Antropología Chilena 34:253-263.

Aufderheide, A, W. Salo, M. Madden, J. Streitz, J. Buikstra, F. Guhl, B. Arriaza, C. Renier, L. Wittmers Jr. y M. Allison 2004. A 9,000-Year Record of Chagas' Disease. Proceedings of the National Academy of Sciences 101(7):2034-2039.

Aufderheide, A. y C. Rodríguez-Martín 1998. The Cambridge Encyclopedia of Human Paleopathology. Cambridge University Press, Cambridge.

Ballester, B. y F. Gallardo 2011. Prehistoric and historic networks on the Atacama Desert coast (northern Chile). Antiquity $85: 875-889$

Ballester, B., A. San Francisco y F. Gallardo 2010. Modo de vida y economía doméstica de las comunidades cazadoras recolectoras costeras del desierto de Atacama en tiempos coloniales y republicanos. Taltalia 3:21-32.

Bass, W. 1987. Human Osteology: A Laboratory and Field Manual. Special Publication $\mathrm{N}^{\circ}$ 2. Missouri Archaeological Society, Columbia.

Berdichewsky, B. 1962. El precerámico de Taltal y sus correlaciones. Centro de Estudios Antropológicos 16.

Bird, J. 1943. Excavations in Northern Chile. Anthropological Papers of the American Museum of Natural History 38.

- - - 1946. The Cultural sequence of the North Chilean coast. En Handbook of South American Indians, editado por J. Steward, pp. 587-597. Bureau of American Ethnology Bulletin 143, Smithsonian Institution, Washington.
Bittmann, B. 1979. Cobija y sus alrededores en la época colonial (1600-1750). Actas del VII Congreso de Arqueología de Chile, Vol. II, pp. 327-64. Ediciones Kultrún, Santiago.

Bombellini R. 1976. Osteoarthritis of the hip. Pathogenisis and Consequent Therapy. Springer-Verlag, Berlín.

Boston, C. y B. Arriaza 2009. Arseniasis and teratogenic anomalies in the Atacama Desert coast of ancient Chile. Interciencia 34(5):338-343.

Buikstra, J. y D. Ubelaker (eds.) 1994. Standards for the Data Collection from Human Skeletal Remains. Arkansas Archaeological Survey Research Series 44, Fayetteville.

Campillo, D. y M.E. Subirà 2004. Antropología Física para Arqueologos. Ariel Prehistoria, Barcelona.

Capdeville, A. 1921. Notas Acerca de la Arqueología de Taltal. Boletín de la Academia Nacional de Historia II(3-4):1-23.

- - - 1922. Notas acerca de la Arqueología de Taltal. Boletín de la Academia Nacional de Historia III(7-8):1-9.

- - - 1923. Un centenario Chincha-Atacameño de Punta Grande, Taltal. Boletín de la Academia Nacional de Historia VII:34-49.

Castelleti, J. 2007. Patrón De Asentamiento y Uso de Recursos a Través de la Secuencia Ocupacional Prehispana En la Costa de Taltal. Tesis para optar al grado de Magister en Antropología con mención en Arqueología,Universidad Católica del NorteUniversidad de Tarapacá, San Pedro de Atacama-Arica.

Castelleti, J. y G. Maltrain 2010. El Formativo de Taltal y el patrón de asentamiento local. Actas del XVII Congreso Nacional de Arqueología Chilena, Vol. 1, pp. 165-176. Ediciones Kultrún, Valdivia.

Castelleti, J., O. Reyes, G. Maltrain, I. Martínez, P. Galarce, H. Velásquez y J.P. Ugalde 2010. Ocupaciones en abrigos rocosos en la costa de Taltal: patrón de uso del espacio desde momentos holocénicos tempranos. Actas del XVII Congreso Nacional de Arqueología Chilena, Vol. 2, pp. 685-695. Ediciones Kultrún, Valdivia.

Castillo, G. 1986. Sociedades agropecuarias tempranas y control de recursos en el ambiente semiárido de Chile. Chungara Revista de Antropología Chilena 16-17:119-124.

Cocilovo, J.A., S. Quevedo, H.H. Varela, S. Valdano y M. Castro 1999. Biología del grupo prehistórico de Pisagua, costa norte de Chile. Estudios Atacameños 17:207-235.

Cocilovo, J.A, H.H. Varela, M.A. Costa-Junqueira y S. Quevedo 2005. Los pescadores arcaicos de la desembocadura del río Loa (norte de Chile): el sitio Caleta Huelén 42. Chungara Revista de Antropología Chilena 37:5-19.

Cohen, M.N. y G.J. Armelagos (eds.) 1984. Paleopathology at the Origins of Agriculture. Academic Press, Orlando.

Contreras, R., J. Cruz, A. Llagostera, H. Garcés, P. Núñez, O. Rodríguez, H. Gárate y G. Becerra 2008. Los Bronces-1: un asentamiento de 5.500 años en la costa de Taltal. Taltalia 1:61-74.

Contreras, R. y P. Núñez 2009. Nuevos antecedentes sobre la balsa de cuero de lobo en la costa de Taltal, Chile. Taltalia 2:88-97.

Contreras, R., P. Núñez, A. Llagostera, J. Cruz, A. San Francisco, B. Ballester, O. Rodríguez y G. Becerra 2011. Un conglomerado del período Arcaico costero medio del área Taltal Paposo, Norte de Chile. Taltalia 4:7-31. 
Constantinescu, F. 2001. Canal Maule y Punta Santa Ana: el habitus cazador recolector marítimo femenino (patrones óseos característicos del modo de vida canoero en el extremo sur de Chile). Anales del Instituto de la Patagonia 29:163-181.

Costa-Junqueira, M.A., J. Cocilovo y S .Quevedo 2000. Patologías óseas, traumas y otros atributos en el grupo Arcaico de Morro de Arica, norte de Chile. Chungara Revista de Antropología Chilena 32:79-83.

Costa-Junqueira, M.A., H. Varela, J. Cocilovo, S. Quevedo y S. Valdano 2000. Perfil paleodemográfico de Morro de Arica, una población de pescadores arcaicos del norte de Chile. Boletín del Museo Nacional de Historia Natural 49:215-235.

Cybulski, J.S. 1977. Cribra Orbitalia, a possible sign of anemia in early historic native populations of the British Columbia Coast. American Journal of Physical Anthropology 47:31-40.

Del Ángel, A.E. y H.B. Cisneros 2002. Modificación de las ecuaciones de regresión para estimar estatura elaboradas por $\mathrm{S}$. Genovés. Boletín de la Asociación Mexicana de Antropología Biológica. Disponible en http://www.geocities.com/amabmex/ publicaciones/boletin/agosto2002.html\#modifica (2005)

Durán, A. 1985. Algunos problemas en relación a las ocupaciones con anzuelo de concha en la zona de Taltal. Actas del IX Congreso Nacional de Arqueología Chilena, pp. 105-125. Museo Arqueológico de La Serena, La Serena.

Ferreira, L., A. de Araujo, U. Confalonieri y L. Núñez. 1984. The finding of eggs of Diphyllobothrium in human coprolites (4,100-1,950 B.C.) from northern Chile. Memorias do Instituto Oswaldo Cruz 79:175-180.

Gaete, N. 2001. Informe de caracterización del sitio 02 Ta 012 "Camarones". Manuscrito en posesión del autor.

Henríquez, M. y B. Arriaza 2013. Distribución y frecuencia de nódulos de Schmörl en la columna vertebral de poblaciones prehispánicas de Arica: ¿indicadores de la carga laboral? Chungara Revista de Antropología Chilena 45:311-319.

Hershkovitz, I., B. Latimer, O. Dutour, L. Jellema, S. WishBaratz, C. Rothschild y B. Rothschild 1997. Why do we fail in aging the skull from the sagittal suture? American Journal of Physical Anthropology 103:393-399.

Hummert, J.R. y D.P. Van Gerven 1985. Observations on the formation and persistance of radiopaque transverse lines. American Journal of Physical Anthropology 66: 297-306.

Iscan, M.Y., S.R. Loth y R.K. Wright 1984. Metamorphosis at sternal rib end: A new method to estimate age at death in white males. American Journal of Physical Anthropology 65:147-156.

Joyce, R.A. 2005. The archaeology of the body. Annual Reviews in Anthropology 34:139-158.

Jurmain, R.D. y L. Kilgore 1995. Skeletal evidence of osteoarthritis: a paleopathological perspective. Annals of Rheumatoid Disease 54:443-450.

Keenleyside, J. 1998. Skeletal evidence of health and disease in pre-contact Alaskan Eskimos and Aleuts. American Journal of Physical Anthropology 107:51-70.

Kennedy, K.A.R. 1989. Skeletal markers of occupational stress. En Reconstruction of Life from the Skeleton, editado por M.Y. Iscan y K.A.R. Kennedy, pp. 129-160. Alan R. Liss, New York.
Knüsel, C. 2000. Bone adaptation and its relationship to physical activity in the past. En Human Osteology and Forensic Science, editado por M. Cox y S. Mays, pp. 381-402. Cambdrige University Press, Cambrige.

Kuzmanic, I. y G. Castillo. 1986. Estadio Arcaico en la costa del norte semiárido de Chile. Chungara Revista de Antropologpia Chilena 16/17:89-94.

Larsen, C.S. 1995. Biological changes in human populations with agriculture. Annual Reviews of Anthropology 24:185-213.

_. - _ 1997. Bioarchaeology. Interpreting Behavior from the Human Skeleton. Cambrige University Press, Cambridge.

Lewis, M.E. 2007. The Bioarchaeology of Children. Perspectives from Biological and Foresinc Anthropology. Cambridge University Press, Cambridge.

Llagostera, A. 1989. Caza y pesca marítima (9.000 a 1.000 a. C.). En Culturas de Chile. Prehistoria. Desde sus Orígenes hasta los Albores de la Conquista, editado por J. Hidalgo, V. Schiappacasse, H. Niemeyer, C. Aldunate e I. Solimano, pp. 57-79. Editorial Andrés Bello, Santiago.

- . - 2005. Culturas costeras precolombinas en el norte chileno: secuencia y subsistencia de las poblaciones arcaicas. En Biodiversidad Marina: Valoración, Usos, Perspectivas ¿Hacia dónde va Chile?, editado por E. Figueroa, pp. 107-148. Editorial Universitaria, Santiago.

Llagostera, A y V. Llagostera 2010. Enterratorios del sitio arqueológico Los Bronces-1, Comuna de Taltal (Región de Antofagasta). Taltalia 3:7-20.

Malgosa, A. 2003. Marcadores de estrés ocupacional. En Paleopatología. La Enfermedad no Escrita, editado por A. Isidro y A. Malgosa, pp. 221-236. Masson, Barcelona.

Mann, R.W. y D.R. Hunt 2005. Photographic Regional Atlas of Bone Disease. A Guide to Pathologic and Normal Variation in the Human Skeleton. Charles C. Thomas Publisher, Springfield.

Marquet, P.A., C.M. Santoro, C. Latorre, V.G. Standen, S.R. Abades, M.M. Rivadeneira, B. Arriaza y M.E. Hochberg 2012. Emergence of social complexity among coastal hunter-gatherers in the Atacama Desert of northern Chile. Proceedings of the National Academy of Sciences 109:14754-14760.

Meskell, L. 1998. The Irresistible Body and Seduction of Archaeology. En Changing Bodies, Changing Meanings: Studies on the Human Body in Antiquity, editado por D. Montserrat, pp. 139-161. Routledge, New York.

Molnar, P. 2006. Tracing prehistoric activities: musculoskeletal stress marker analysis of a Stone-Age population on the Island of Gotland in the Baltic sea. American Journal of Physical Anthropology 129:12-23.

Mostny, G. 1964. Arqueología de Taltal. Epistolario de Augusto Capdeville con Max Uhle y Otros. Fondo Histórico y Bibliográfico José Toribio Medina, Santiago.

Muñoz, I. 1989. El Período Formativo en el Norte Grande (100 a.C. a 500 d. C.). En Culturas de Chile. Prehistoria. Desde sus orígenes hasta los albores de la Conquista, editado por J. Hidalgo et al., pp. 107-128. Editorial Andrés Bello, Santiago.

Muñoz, I. y J. Chacama 2012. Transformación del paisaje social en Arica, norte de Chile: De pescadores arcaicos a agricultores incipientes. Estudios Atacameños 44:123-140. 
Niemeyer, H. 1997. El período temprano del horizonte agroalfarero en Copiapó. En Culturas Prehistóricas de Copiapó, editado por H. Niemeyer, M. Cervellino y G. Castillo, pp. 39-60. Museo Regional de Atacama, Copiapó.

Núñez, L. 1984. Secuencia de asentamientos prehistóricos del área de Taltal. Revista Futuro 8:28-76.

Núñez, L. y H. Hall. 1982. Análisis de dieta y movilidad en un campamento arcaico del norte de Chile. Boletín del Instituto Francés de Estudios Andinos 3-4: 91-113.

Núñez, L., y C. Santoro, 2011. El tránsito Arcaico-Formativo en la Circumpuna y Valles Occidentales del Centro-Sur andino: Hacia los cambios "neolíticos". Chungara Revista de Antropología Chilena 43:487-530.

Núñez, P. 2003. Vivir el Mar. Ediciones de la Universidad de Antofagasta, Antofagasta.

Olguín, L., D. Salazar y D. Jackson 2014. Tempranas evidencias de navegación y caza de especies oceánicas en la costa pacífica de Sudamérica (Taltal, 7.000 años cal. a.p.). Chungara Revista de Antropología Chilena 46(2), en prensa.

Petruzelli, B., A. Roberts, D. Pate, C. Santoro, T. Maddern, C. Carter y M. Westaway 2012. Stable carbón and nitrogen isotopic analysis of skeletal remains from Azapa 71 and Pica-8, northern Chile: an assessment of human diet and landscape use in the Late Holocene. Journal of the Anthropological Society of South Australia 35:52-80.

Quevedo, S. 1998. Biología de una Población Arcaica del Norte Semiárido Chileno. Tesis Doctoral, Facultad de Filosofía y Letras, Universidad de Buenos Aires, Buenos Aires.

- _ _ 2000. Patrones de actividad a través de las patologías en población arcaica de Punta Teatinos, Norte Semiárido Chileno. Chungara Revista de Antropología Chilena 32:11-21.

Quevedo, S. y J.A. Cocilovo 1995. La población antigua de Pisagua. Caracterización bioantropológica del cementerio " $\mathrm{C}$ " de la colección Max Uhle. En Nuestros Viejos Planteamientos para Nuevas Preguntas, Nuestros Viejos Huesos para Nuevas Respuestas: Cambio Cultural es Cambio Biologico? Informe Final Proyecto FONDECYT 1950218. Manuscrito en posesión del autor.

Quevedo, S., J. Cocilovo, M. Costa, H. Varela y S. Valdano 2000. Perfil paleodemográfico de Punta Teatinos. Una población de pescadores arcaicos del norte semiárido. Boletín del Museo Nacional de Historia Natural 49:237-256.

Quevedo, S., J. Cocilovo, H. Varela y M.A. Costa-Junqueira 2003. Perfil Paleodemográfico del El Cerrito (La Herradura), un Grupo de Pescadores Arcaicos del Norte Semiárido de Chile. Boletín del Museo Nacional de Historia Natural 52:177-194.

Reinhard, K y O. Urban 2003. Diagnosing ancient diphyllobothriasis from Chinchorro mummies. Memorias do Instituto Oswaldo Cruz 98(1):191-193.

Rhodes, J. 2006. Adaptations to humeral torsion in medieval Britain. American Journal of Physical Anthropology 130:106-166.

Rhodes, J. y S. Churchill 2008. Throwing in the Middle and Upper Paleolithic: inferences from an analysis of humeral retroversion. Journal of Human Evolution 56:1-10.
Rivera, M. 2002. Historias del Desierto, Arqueología del Norte de Chile. Editorial del Norte, La Serena.

Roberts, A., D. Pate, B. Petruzzelli, C. Carter, M. Westaway, C. Santoro, J. Swift, T. Maddern, G. Jacobsen, F. Bertuch y F. Rothhammer 2013. Retention of hunter-gatherer economies among maritime foragers from Caleta Vitor, northern Chile, during the late Holocene: evidence from stable carbon and nitrogen isotopic analysis of skeletal remains. Journal of Archaeological Science 40:2360-2372.

Roberts, C. 2000. Infectious disease in biocultural perspective: past, present and future work in Britain. En Human Osteology in Archaeological and Forensic Science, editado por M. Cox y S. Mays, pp. 145-162. Cambridge University Press, New York.

Roberts, C. y K. Manchester 2005. The Archaeology of Disease. Sutton Publishing, Gloucesershire.

Rothschild, B. 2000. Porotic hyperostosis as a manifestation of iron deficiency? Chungara Revista de Antropología Chilena 32:85-87.

Salazar, D. 2013. Aproximación preliminar a la cronología, asentamiento y organización económica de las poblaciones arcaicas de la costa de Taltal. Anexo 1, Informe de Avance Año 2, Proyecto Fondecyt 1110196. Manuscrito en posesión del autor.

Salazar, D., P. Andrade, C. Borie, M. Escobar, V. Figueroa, C. Flores, L. Olguín y H. Salinas 2013. Nuevos sitios correspondientes al Complejo Cultural Huentelauquén en la costa de Taltal. Taltalia 5-6:9-19.

Salazar, D., V. Castro, H. Salinas y V. Varela 2009. Nuevas investigaciones sobre la prehistoria y la antigua minería de Taltal. Taltalia 2:111-118.

Salazar, D., D. Jackson, J.L. Guendon, H. Salinas, D. Morata, V. Figueroa, G. Manríquez y V. Castro 2011. Early evidence (ca. 12,000 BP) for iron oxide mining on the Pacific coast of South America. Current Anthropology 52:463-475.

Sandweiss, D. 2008. Early fishing societies in western South America. En Handbook of South American Archaeology, editado por H. Silverman y W.H. Isbell, pp. 145-156. Springer, New York.

Santoro, C., M. Rivadeneira, C. Latorre, F. Rothhammer y V. Standen 2012. Rise and decline of Chinchorro sacred landscapes along the hyperarid coast of the Atacama desert. Chungara Revista de Antropología Chilena 44 (4): 637-653.

Santos, F.L.N. y L.B. de Faro 2005. The first confirmed case of Diphyllobothrium latum in Brazil. Memorias do Instituto Oswaldo Cruz 100(6):585-586.

Scheuer, L. y S. Black 2000. Developmental Juvenile Osteology. Academic Press, San Diego.

Silva, J. y D. Bahamondes 1969. Investigaciones arqueológicas en Taltal, Informe Preliminar. Actas del IV Congreso Nacional de Arqueología Chileno Rehue 2:7-25.

Sisson, T. y C. Lund 1958. The influence of maternal iron deficiency on the newborn. The American Journal of Clinical Nutrition 6:376-385.

Sofaer, J.R. 2006. The Body as Material Culture. A Theoretical Osteoarchaeology. Cambridge University Press, Cambridge. 
Standen, V., M. Allison y B. Arriaza 1984. Patologías óseas de la población Morro-1: asociada al complejo Chinchorro: Norte de Chile. Chungara Revista de Antropología Chilena 13:175-185.

Standen, V.G. y B.T. Arriaza 2000. La Treponematosis prehispánica en el norte de Chile. Chungara Revista de Antropología Chilena 32:185-192.

Standen, V., B. Arriaza y C. Santoro 1995. Una hipótesis ambiental para un marcador óseo: la exostosis auditiva externa en las poblaciones humanas prehistóricas del desierto del norte de Chile. Chungara Revista de Antropología Chilena 27:99-116.

- - - 1997. External auditory exostosis in prehistoric chilean populations: a test of the cold water hipótesis. American Journal of Physical Anthropology 103:119-129.

Storey, R., L. Marquez Morfin y V. Smith 2002. Social disruption and the maya civilization of Mesoamerica: a study of health and economy of the last thousand years. En The Backbone of History. Health and Nutrition in the Western Hemisphere, editado por R.H. Steckel y J.C. Rose, pp. 283-306. Cambridge University Press, Cambridge.

Stuart -Macadam, P. 1989. Nutritional deficiency diseases: a survey of scurvy, rickets and iron-deficiency anemia. En Reconstruction of Life from the Skeleton, editado por M.Y Iscan y K.A.R. Kennedy, pp. 201-222. Alan L. Riss, New York.

- - - 2005. Iron deficiency anemia: exploring the difference. En Sex and Gender in Paleopathological Perspective, editado por A.L. Grauer y P. Stuart -Macadam, pp. 45-63. Cambridge University Press, New York.

Synnott, A. 1993. The Body Social: Symbolism, Self, and Society. Routledge, Londres.
Trinkaus, E. 1975. Squatting among the Neanderthal: a problem in the behavioral interpretation of skeletal morphology. Journal of Archaeological Science 2:327-351.

Troncoso, A. y D. Pavlovic 2013. Historia, saberes y prácticas: un ensayo sobre el desarrollo de las comunidades alfareras del Norte Semiárido chileno. Revista Chilena de Antropología 27 (1):101-140.

Walker, P.L. 1985. Anemia among prehistoric indians of the american southwest. En Health and Disease in the Prehistoric Southwest, editado por C.F. Merbs y R.J. Miller, pp. 139-162. Arizona State University, Arizona.

- - - 1986. Porotic hyperostosis in a marine - dependent California indian population. American Journal of Physical Anthropology 69:345-354.

Watson, J.T., B. Arriaza, V. Standen e I. Muñoz 2013. Tooth wear related to marine foraging, agro-pastoralism and the Formative transition on the Northern Chilean coast. International Journal of Osteoarchaeology 23:287-302.

Weston, D.A. 2008. Investigating the specificity of periosteal reactions in pathology museum specimens. American Journal of Physical Anthropology 137:48-59.

White, C., J. Maxwell, A. Dolphin, J. Williams y F. Longstaffe 2006. Paleoecology and paleodiet in postclassic/historic maya from northern coastal Belize. Memorias do Instituto Oswaldo Cruz 101(II):35-42.

Wood, J.W., G.R. Milner, H.C. Harpending y K.M. Weiss 1992. The osteological paradox: problems of inferring prehistoric health from skeletal samples. Current Anthropology 33:343-370.

Yesner, D. 1980. Maritime hunter-gatherers: Ecology and Prehistory. Current Anthropology 21:727-751.

\section{Notas}

1 Investigación realizada dentro del marco de los proyectos FONDECYT 1080666 y 1110196.
2 Excavados en el marco de los proyectos FONDECYT 1080666 y 1100196, así como de Estudios de Impacto Ambiental realizados previamente en al área (Castelleti 2007). 
